\title{
Conformally Equivariant Quantization - a Complete Classification
}

\author{
Jean-Philippe MICHEL \\ University of Luxembourg, Campus Kirchberg, Mathematics Research Unit, \\ 6, rue Richard Coudenhove-Kalergi, L-1359 Luxembourg City, Luxembourg \\ E-mail: jean-philippe.michel@uni.lu \\ URL: http://math.uni.lu/ michel/SitePerso/indexLux.html
}

Received July 29, 2011, in final form April 11, 2012; Published online April 15, 2012

http://dx.doi.org/10.3842/SIGMA.2012.022

\begin{abstract}
Conformally equivariant quantization is a peculiar map between symbols of real weight $\delta$ and differential operators acting on tensor densities, whose real weights are designed by $\lambda$ and $\lambda+\delta$. The existence and uniqueness of such a map has been proved by Duval, Lecomte and Ovsienko for a generic weight $\delta$. Later, Silhan has determined the critical values of $\delta$ for which unique existence is lost, and conjectured that for those values of $\delta$ existence is lost for a generic weight $\lambda$. We fully determine the cases of existence and uniqueness of the conformally equivariant quantization in terms of the values of $\delta$ and $\lambda$. Namely, (i) unique existence is lost if and only if there is a nontrivial conformally invariant differential operator on the space of symbols of weight $\delta$, and (ii) in that case the conformally equivariant quantization exists only for a finite number of $\lambda$, corresponding to nontrivial conformally invariant differential operators on $\lambda$-densities. The assertion (i) is proved in the more general context of IFFT (or AHS) equivariant quantization.
\end{abstract}

Key words: quantization; (bi-)differential operators; conformal invariance; Lie algebra cohomology

2010 Mathematics Subject Classification: 53A55; 53A30; 17B56; 47E05

\section{Introduction}

Quantization originates from physics, and lies at the correspondence between classical and quantum formalisms. It has given the impetus for the development of numerous mathematical theories, and admits as many different definitions. In this paper, by quantization we mean the inverse of a certain symbol map, i.e. a linear map from functions on a cotangent bundle $T^{*} M$, which are polynomial in the fiber variables, to the space of differential operators on $M$. No such quantization can be canonically defined from the differential geometry of $M$. The idea of equivariant quantization is to build one from a richer geometric structure on $M$ : the local action of a Lie group $G$, or equivalently, of its Lie algebra $\mathfrak{g}$. This imposes the condition that the manifold $M$ possesses a locally flat $G$-structure.

Equivariant quantization was first developed for projective and conformal structures by Duval, Lecomte and Ovsienko [7, 17]. There, the condition of equivariance singles out a unique quantization up to normalization. Generalizations to the curved case have been proposed recently in terms of Cartan connections $[19,20]$ or tractors $[6,23]$, but we restrict ourselves here to the locally flat case, or equivalently to $\mathbb{R}^{n}$. In [4], Boniver and Mathonet exhibit the correct class of Lie algebras to be considered for equivariant quantization. It is provided by maximal Lie subalgebras $\mathfrak{g}$ among those of finite dimension in $\operatorname{Vect}_{\text {pol }}\left(\mathbb{R}^{n}\right)$, the Lie algebra of polynomial vector fields. They prove in [5] that these Lie algebras are precisely the irreducible filtered Lie algebras of finite type (with no complex structure) classified by Kobayashi and Nagano [13], referred to as IFFT-algebras. Particular examples are the Lie algebra $\mathfrak{s l}_{n+1} \simeq \mathfrak{s l}(n+1, \mathbb{R})$ of projective vector 
fields, and the Lie algebra $\mathfrak{c} \mathfrak{f} \simeq \mathfrak{o}(p+1, q+1)$ of conformal Killing vector fields on $\left(\mathbb{R}^{n}, \eta\right)$, where $n=p+q$ and $\eta$ is the flat metric of signature $(p, q)$. Generalizing [4], Cap and Silhan prove in [6] the existence and uniqueness of the $\mathfrak{g}$-equivariant quantization with values in differential operators acting on irreducible homogeneous bundles, barring certain exceptional bundles. The determination of these exceptional cases and their geometric interpretation is an open problem, since they appear in the seminal work [7] dealing with bundles of tensor densities $\left|\Lambda^{n} T^{*} \mathbb{R}^{n}\right|^{\otimes \lambda}$ of arbitrary weight $\lambda \in \mathbb{R}$. Our paper is devoted to a step towards its solution. We give a complete resolution in the original case of conformally equivariant quantization $\mathcal{Q}^{\lambda, \mu}: \mathcal{S}^{\delta} \rightarrow \mathcal{D}^{\lambda, \mu}$ with values in the space $\mathcal{D}^{\lambda, \mu}$ of differential operators from $\lambda$ - to $\mu$-tensor densities, the symbols being here of weight $\delta=\mu-\lambda$.

This work can be regarded in the broader perspective of invariant bidifferential operators, whose equivariant quantization is an example. Another example is provided by generalized transvectants (or Rankin-Cohen bracket), whose arguments are spaces of weighted densities. There the same phenomenon occurs: they exist and are unique except for certain exceptional weights [22]. In fact, this is general, as proved by Kroeske in his thesis [14], where he studies invariant bidifferential operators for parabolic geometries. From a number of examples and a proof in the projective case [15], Kroeske proposes a paradigm which can be roughly phrased as follows: every exceptional case of existence or uniqueness of an invariant bidifferential operator originates from the existence of a nontrivial invariant differential operator on one of the factors. We show that this is particularly illuminating in the case of equivariant quantization. Namely, resorting to the interpretation in terms of cohomology of $\mathfrak{g}$-modules developed by Lecomte in [16], we prove our first main result: unique existence of $\mathfrak{g}$-equivariant quantization is lost if and only if there exists a $\mathfrak{g}$-invariant operator on the space of symbols. Remarkably, this correspondence is proved directly rather than obtained à posteriori, like in the work of Kroeske. The theory of invariant operators being well-developed, we get an efficient way to determine the exceptional bundles for equivariant quantization. Returning to the aforementioned conformally equivariant quantization $\mathcal{Q}^{\lambda, \mu}: \mathcal{S}^{\delta} \rightarrow \mathcal{D}^{\lambda, \mu}$, we recover the set of critical values of $\delta$ for which unique existence is lost, already determined by Silhan [23].

The next natural question is to determine for which resonant pairs of irreducible homogeneous bundles the g-equivariant quantization exists but is not unique. This turns to be an harder question, and we address it only for pairs of line bundles of tensor densities. By our first main theorem, we can obtain the critical shift $\delta=\mu-\lambda$ of their weights for which there is not existence and uniqueness of the $\mathfrak{g}$-equivariant quantization. We have then to determine the resonant values $(\lambda, \mu)$ for which the $\mathfrak{g}$-equivariant quantization still exists. In the projective case, an explicit formula for the quantization provides the answer [9, 17]. Moreover, the situation has been fully understood in terms of cohomology of $\mathfrak{s l}_{n+1}$-modules, the existence of the projectively equivariant quantization being characterized by the triviality of a 1-cocycle, which depends on $\delta$ and $\lambda$ [16]. Using a similar approach, we obtain our second main result: for each critical value $\delta$ of the conformally equivariant quantization $\mathcal{Q}^{\lambda, \mu}$, there is a finite number of resonances $(\lambda, \lambda+\delta)$ that we determine. This completes the known results on symbols of degree at most 3 , and proves a conjecture of Silhan: the conformally equivariant quantization does generically not exist for $\delta$ critical. In addition, we provide an interpretation along the lines of the Kroeske's paradigm: the resonances correspond to conformally invariant operators on the source space of densities, e.g. the conformal powers of the Laplacian. This allows us to construct conformally equivariant quantization in the resonant cases. Together with Silhan's work [23], this provides construction for the conformally equivariant quantization whenever it exists.

Let us outline the contents of this paper. Section 2 is devoted to the proof of Theorem 2.5: for $\mathfrak{g}$ an IFFT-algebra, the $\mathfrak{g}$-equivariant quantization exists and is unique if and 
only if there is no $\mathfrak{g}$-invariant differential operator on the space of symbols which strictly lowers the degree. It relies on the fact that equivariant quantization exists if a certain 1-cocycle is a coboundary. We know from [4] that this is the case for generic $\delta^{\prime}$, and this property is stable in the limit $\delta^{\prime} \rightarrow \delta$ if the space of 1-coboundaries is of constant dimension. That condition happens to be equivalent to the absence of $\mathfrak{g}$-invariant operators on $\delta$-weighted symbols. In Sections 3 and 4 we illustrate and complete Theorem 2.5 for, respectively, $\mathfrak{g}$ the projective and the conformal Lie algebras, the quantization being valued in differential operators acting on tensor densities. First, we determine the critical values via the classification of invariant operators on the space of symbols. Then we prove that those invariant operators give rise to nontrivial 1-cocycles. They obstruct existence of the equivariant quantization except when there is an invariant differential operator from $\lambda$-densities to a certain homogeneous bundle. Finally, the latter operator allows us to construct an equivariant quantization, proving thus its existence for exactly those values of $\lambda$. That leads to the complete list of resonances $(\lambda, \lambda+\delta)$, together with their interpretation in terms of invariant operators on the space of $\lambda$-densities. We end Section 4 with a detailed treatment of the symbols of degree less than 3, thus interpreting the results of Loubon Djounga [18] along the line of the Kroeske's paradigm. The last section gives us the opportunity to propose some natural extensions of our results.

Throughout this paper, the space of linear maps between two real vector spaces $V$ and $W$ is denoted by $\operatorname{Hom}(V, W)$, and its elements are called operators. We work on $\mathbb{R}^{n}$ and the Einstein summation convention is understood.

\section{On the existence and uniqueness of equivariant quantization}

\subsection{Definition of equivariant quantization}

We start with the definitions of the algebra $\mathcal{D}\left(\mathbb{R}^{n}\right)$ of differential operators on $\mathbb{R}^{n}$, and its algebra of symbols $\mathcal{S}\left(\mathbb{R}^{n}\right)$. The former is filtered by the subspaces $\mathcal{D}_{k}\left(\mathbb{R}^{n}\right)$ of differential operators of order $k$, defined as the spaces of operators $A$ on $\mathcal{C}^{\infty}\left(\mathbb{R}^{n}\right)$ satisfying $\left.\left[\ldots\left[A, f_{0}\right], \ldots\right], f_{k}\right]=0$ for all functions $f_{0}, \ldots, f_{k} \in \mathcal{C}^{\infty}\left(\mathbb{R}^{n}\right)$, considered here as (zero order) operators on $\mathcal{C}^{\infty}\left(\mathbb{R}^{n}\right)$. The latter is the canonically associated graded algebra, defined by $\mathcal{S}\left(\mathbb{R}^{n}\right)=\bigoplus_{k=0}^{\infty} \mathcal{D}_{k}\left(\mathbb{R}^{n}\right) / \mathcal{D}_{k-1}\left(\mathbb{R}^{n}\right)$. This may be identified with the algebra of functions on $T^{*} \mathbb{R}^{n}$ that are polynomial in the fibers, the grading corresponding to the polynomial degree. The canonical projection $\sigma_{k}: \mathcal{D}_{k}\left(\mathbb{R}^{n}\right) \rightarrow$ $\mathcal{D}_{k}\left(\mathbb{R}^{n}\right) / \mathcal{D}_{k-1}\left(\mathbb{R}^{n}\right)$ is called the principal symbol map. It admits a section, the normal ordering, given by

$$
\mathcal{N}: P^{i_{1} \ldots i_{k}}(x) p_{i_{1}} \cdots p_{i_{k}} \mapsto P^{i_{1} \ldots i_{k}}(x) \partial_{i_{1}} \cdots \partial_{i_{k}}
$$

where $\left(x^{i}, p_{i}\right)$ are coordinates on $T^{*} \mathbb{R}^{n}$. This defines a linear isomorphism $\mathcal{S}\left(\mathbb{R}^{n}\right) \simeq \mathcal{D}\left(\mathbb{R}^{n}\right)$.

We are interested in the action of vector fields on these both algebras. First of all, we introduce the $\operatorname{Vect}\left(\mathbb{R}^{n}\right)$-module of $\lambda$-densities $\mathcal{F}^{\lambda}=\left(\mathcal{C}^{\infty}\left(\mathbb{R}^{n}\right), \ell^{\lambda}\right)$ as a one parameter deformation of the module $\mathcal{C}^{\infty}\left(\mathbb{R}^{n}\right)$, the $\operatorname{Vect}\left(\mathbb{R}^{n}\right)$-action being given by $X \mapsto \ell_{X}^{\lambda}=X+\lambda \operatorname{Div}(X)$, with $\operatorname{Div}$ the divergence and $\lambda \in \mathbb{R}$ the weight of the densities. This module corresponds geometrically to the space of sections of the trivial line bundle $\left|\Lambda^{n} T^{*} \mathbb{R}^{n}\right|^{\otimes \lambda}$. It gives rise to the $\operatorname{Vect}\left(\mathbb{R}^{n}\right)$-module $\mathcal{D}^{\lambda, \mu}=\left(\mathcal{D}\left(\mathbb{R}^{n}\right), \mathcal{L}^{\lambda, \mu}\right)$ of differential operators from $\lambda$ - to $\mu$-densities, endowed with the adjoint action

$$
\mathcal{L}_{X}^{\lambda, \mu} A=\ell_{X}^{\mu} A-A \ell_{X}^{\lambda}
$$

for all $X \in \operatorname{Vect}\left(\mathbb{R}^{n}\right)$ and $A \in \mathcal{D}\left(\mathbb{R}^{n}\right)$. This action preserves the filtration of $\mathcal{D}\left(\mathbb{R}^{n}\right)$, and so the algebra of symbols inherits a $\operatorname{Vect}\left(\mathbb{R}^{n}\right)$-module structure compatible with the grading. We 
denote this structure by $\mathcal{S}^{\delta}=\left(\mathcal{S}\left(\mathbb{R}^{n}\right), L^{\delta}\right)$, where $\delta=\mu-\lambda$ is the shift. The action of $\operatorname{Vect}\left(\mathbb{R}^{n}\right)$ is given in coordinates by

$$
L_{X}^{\delta}=X^{i} \partial_{i}-p_{j}\left(\partial_{i} X^{j}\right) \partial_{p_{i}}+\delta \operatorname{Div}(X),
$$

and coincides with the canonical action on functions on $T^{*} M$ tensored with $\delta$-densities. We denote by $\mathcal{S}_{k}^{\delta}$ the submodule of homogeneous symbols of degree $k$.

Definition 2.1. Let $\mathfrak{g}$ be a Lie subalgebra of $\operatorname{Vect}\left(\mathbb{R}^{n}\right)$. A $\mathfrak{g}$-equivariant quantization is a $\mathfrak{g}$ module morphism

$$
\mathcal{Q}^{\lambda, \mu}: \mathcal{S}^{\delta} \rightarrow \mathcal{D}^{\lambda, \mu}
$$

such that $\mathcal{Q}^{\lambda, \mu}$ is a right inverse of the principal symbol map on homogeneous symbols.

Using the normal ordering (2.1), we freely identify $\mathcal{D}^{\lambda, \mu}$ with $\left(\mathcal{S}\left(\mathbb{R}^{n}\right), \mathcal{L}^{\lambda, \mu}\right)$, where we keep the same notation for $\mathcal{L}^{\lambda, \mu}$ and its pull-back on symbols by $\mathcal{N}$. It is then a matter of computation to prove that $\mathcal{L}_{X}^{\lambda, \mu}=L_{X}^{\delta}$ if $X$ is an affine vector field and $\delta=\mu-\lambda$. In other words, $\mathcal{N}$ is an $\mathfrak{a} \mathfrak{f} \mathfrak{f}(n, \mathbb{R})$-equivariant quantization [7].

\subsection{IFFT-algebras and equivariant quantization}

An IFFT-algebra $\mathfrak{g}$ is a simple Lie subalgebra of the polynomial vector fields on $\mathbb{R}^{n}$. As such, it admits a gradation by the degree of the vector field components $\mathfrak{g}=\mathfrak{g}_{-1} \oplus \mathfrak{g}_{0} \oplus \mathfrak{g}_{1}$ which is compatible with the bracket: $\left[\mathfrak{g}_{i}, \mathfrak{g}_{j}\right]=\mathfrak{g}_{i+j}$, where $\mathfrak{g}_{i}=\{0\}$ if $i \notin\{-1,0,1\}$. The Lie subalgebra $\mathfrak{g}_{-1}$ consists of translations, $\mathfrak{g}_{1}$ consists of so-called $\mathfrak{g}$-inversions, and $\mathfrak{g}_{0}$ contains the dilation and acts irreducibly on both $\mathfrak{g}_{-1}$ and $\mathfrak{g}_{1}$. Consequently, $\mathfrak{g}$-invariance is equivalent to $\mathfrak{g}_{-1} \oplus \mathfrak{g}_{0}$-invariance plus the invariance with respect to one non-zero element in $\mathfrak{g}_{1}$.

Let us introduce some notation. For $\mathfrak{h}$ a Lie subalgebra of $\operatorname{Vect}\left(\mathbb{R}^{n}\right)$, the space of $\mathfrak{h}$-invariant operators between two $\mathfrak{h}$-submodules $F \subset \mathcal{S}^{\delta}$ and $F^{\prime} \subset \mathcal{S}^{\delta^{\prime}}$ is defined as

$$
\operatorname{Hom}_{\mathfrak{h}}\left(F, F^{\prime}\right)=\left\{A \in \operatorname{Hom}\left(F, F^{\prime}\right) \mid \forall X \in \mathfrak{h},\left[L_{X}^{*}, A\right]=0\right\},
$$

where the commutator $\left[L_{X}^{*}, A\right]$ is a symbolic notation for $L_{X}^{\delta^{\prime}} A-A L_{X}^{\delta}$. Note that the vector fields which preserve $F$ and $F^{\prime}$ clearly act on $\operatorname{Hom}_{\mathfrak{h}}\left(F, F^{\prime}\right)$. We define similarly the space of $\mathfrak{h}$-invariant differential operators $\mathcal{D}_{\mathfrak{h}}\left(F, F^{\prime}\right)$ and its module structure.

Lemma 2.2 ([17]). Let $\mathfrak{g}$ be an IFFT-algebra, and fix $l<k$. The vector space $\operatorname{Hom}_{\mathfrak{g}_{-1} \oplus \mathfrak{g}_{0}}\left(\mathcal{S}_{k}^{\delta}, \mathcal{S}_{l}^{\delta}\right)$ is finite-dimensional, independent of $\delta$, and equal to the space $\mathcal{D}_{\mathfrak{g}_{-1} \oplus \mathfrak{g}_{0}}\left(\mathcal{S}_{k}^{\delta}, \mathcal{S}_{l}^{\delta}\right)$ of invariant differential operators.

Proof. We only need the fact that $\mathfrak{g}_{-1} \oplus \mathfrak{g}_{0}$ contains the translations and the dilation. Indeed, an operator $A \in \operatorname{Hom}\left(\mathcal{S}_{k}^{\delta}, \mathcal{S}_{l}^{\delta}\right)$ invariant with respect to those transformations was proved in [17] to be a differential operator if $l<k$. Hence it is generated by the coordinates $p_{i}$ and the derivatives $\partial_{i}, \partial_{p_{i}}$. Now the dilation invariance ensures that the degree in $\partial_{i}$ is equal to the degree in $\partial_{p_{i}}$ minus the degree in $p_{i}$. The degree in $\partial_{p_{i}}$ being $k$ at most, the space $\operatorname{Hom}_{\mathfrak{g}_{-1} \oplus \mathfrak{g}_{0}}\left(\mathcal{S}_{k}^{\delta}, \mathcal{S}_{l}^{\delta}\right)$ is finite-dimensional. Since the divergence of affine vector fields is a constant, the action of $\mathfrak{g}_{-1} \oplus \mathfrak{g}_{0}$ on $\operatorname{Hom}\left(\mathcal{S}_{k}^{\delta}, \mathcal{S}_{l}^{\delta}\right)$ is independent of $\delta$ and hence the subspace of $\mathfrak{g}_{-1} \oplus \mathfrak{g}_{0}$-invariant operators is also. 
We turn now to the study of the $\mathfrak{g}$-equivariance condition for a quantization $\mathcal{Q}^{\lambda, \mu}$. We can always factor the latter through the normal ordering, defining the linear automorphism $Q^{\lambda, \mu}$ of $\mathcal{S}^{\delta}$ by $\mathcal{Q}^{\lambda, \mu}=\mathcal{N} \circ Q^{\lambda, \mu}$. Restricting to $\mathcal{S}_{k}^{\delta}$, we then get

$$
Q^{\lambda, \mu}=\sum_{l=0}^{k} \phi_{l},
$$

where $\phi_{0}=\operatorname{Id}$ and $\phi_{l} \in \operatorname{Hom}\left(\mathcal{S}_{k}^{\delta}, \mathcal{S}_{k-l}^{\delta}\right)$. Since the normal ordering is $\mathfrak{a f f}(n, \mathbb{R})$-equivariant, we deduce from Lemma 2.2 that more precisely $\phi_{l} \in \mathcal{D}_{\mathfrak{g}_{-1} \oplus \mathfrak{g}_{0}}\left(\mathcal{S}_{k}^{\delta}, \mathcal{S}_{k-l}^{\delta}\right)$ for all $l=1, \ldots, k$. The full equivariance condition for $\mathcal{Q}^{\lambda, \mu}$ reads then on $\mathcal{S}_{k}^{\delta}$ as

$$
\left[\phi_{l}, L_{X}^{\delta}\right]=\left(\mathcal{L}_{X}^{\lambda, \mu}-L_{X}^{\delta}\right) \phi_{l-1},
$$

for all $l=1, \ldots, k$, where $X$ is a non-zero element of $\mathfrak{g}_{1}$. To solve it directly is too intricate except for $\mathfrak{g}=\mathfrak{s l}_{n+1}$. Nevertheless, the following theorem has been proven in [4], resorting to simultaneous diagonalization of the Casimir operators on the modules of symbols and differential operators. We also refer to [6], where the additional hypothesis of simplicity of the semi-simple part of $\mathfrak{g}_{0}$ is dropped.

Theorem $2.3([4,6])$. Let $\mathfrak{g}$ be an IFFT-algebra and fix $k \in \mathbb{N}$. The $\mathfrak{g}$-equivariant quantization $\mathcal{Q}^{\lambda, \mu}: \mathcal{S}_{k}^{\delta} \rightarrow \mathcal{D}_{k}^{\lambda, \mu}$ exists and is unique if $\mu-\lambda=\delta \notin I_{k}$, with $I_{k}$ a finite subset of $\mathbb{Q}$.

\subsection{Equivariant quantization and Lie algebra cohomology}

We give here a cohomological interpretation of the equations (2.5) encoding the equivariance of $Q^{\lambda, \mu}$. Let us first give a brief review of the cohomology of $\mathfrak{g}$-modules and its link with the splitting of exact sequences of $\mathfrak{g}$-modules (see [12] for more details). The cohomology of the $\mathfrak{g}$-module $\left(M, L^{M}\right)$ is defined in terms of the $k$-cochains, which are the linear maps $\Lambda^{k} \mathfrak{g} \rightarrow M$, and the differential $d$ which reads, on 0 - and 1-cochains $\phi$ and $\gamma$,

$$
d \phi(X)=L_{X}^{M} \phi, \quad d \gamma(X, Y)=L_{X}^{M} \gamma(Y)-L_{Y}^{M} \gamma(X)-\gamma([X, Y]),
$$

where $X, Y$ are in $\mathfrak{g}$. Let now $\left(A, L^{A}\right),\left(B, L^{B}\right),\left(C, L^{C}\right)$ be three $\mathfrak{g}$-modules, and suppose that we have an exact sequence of $\mathfrak{g}$-modules,

$$
0 \longrightarrow\left(A, L^{A}\right) \stackrel{\iota}{\longrightarrow}\left(B, L^{B}\right) \stackrel{\sigma}{\longrightarrow}\left(C, L^{C}\right) \longrightarrow 0
$$

with $\tau$ a linear section. This defines a 1-cocycle $\gamma=\iota^{-1}\left(L^{B} \circ \tau-\tau \circ L^{C}\right)$ with values in $\operatorname{Hom}(C, A)$. Its cohomolgical class does not depend on the choice of linear section $\tau$, and the sequence of $\mathfrak{g}$-modules is split if and only if $\gamma=d \phi$ is a coboundary. The splitting morphism is then $\tau+\iota \circ \phi$. Moreover, if $\gamma$ vanishes on a Lie subalgebra $\mathfrak{h}$ then $\phi$ is $\mathfrak{h}$-invariant.

The existence and uniqueness of a $\mathfrak{g}$-equivariant quantization can be rephrased in terms of cohomology of $\mathfrak{g}$-modules [16]. Indeed, such a quantization exists if for every $k \in \mathbb{N}$ the exact sequence

$$
0 \rightarrow \mathcal{D}_{k-1}^{\lambda, \mu} \rightarrow \mathcal{D}_{k}^{\lambda, \mu} \rightarrow \mathcal{S}_{k}^{\delta} \rightarrow 0
$$

is split in the category of $\mathfrak{g}$-modules. Using the normal ordering as a linear splitting, this means that the 1 -cocycle $\gamma=\mathcal{L}^{\lambda, \mu}-L^{\delta}$ admits a trivial cohomology class $[\gamma]$. By vanishing of $\gamma$ on the affine part of $\mathfrak{g}$, the latter pertains to the following relative cohomology space $H^{1}\left(\mathfrak{g}, \mathfrak{g}_{-1} \oplus \mathfrak{g}_{0} ; \operatorname{Hom}\left(\mathcal{S}_{k}^{\delta}, \mathcal{D}_{k-1}^{\lambda, \mu}\right)\right)$. The modules $\operatorname{Hom}\left(\mathcal{S}_{k}^{\delta}, \mathcal{D}_{k-1}^{\lambda, \mu}\right)$ are quite complex to handle, but 
modding out by $\mathcal{D}_{k-l}^{\lambda, \mu}$ for increasing $l$, we are reduced by induction to the simpler modules $\operatorname{Hom}\left(\mathcal{S}_{k}^{\delta}, \mathcal{S}_{k-l}^{\delta}\right)$. Thus, a g-equivariant quantization on $F^{\delta}$, a submodule of $\mathcal{S}_{k}^{\delta}$, is a section of $\mathfrak{g}$-modules $\psi_{k}: F^{\delta} \rightarrow \mathcal{D}_{k}^{\lambda, \mu}$ defined inductively by $\psi_{0}=$ Id and the commutative triangle in the following diagram of $\mathfrak{g}$-modules

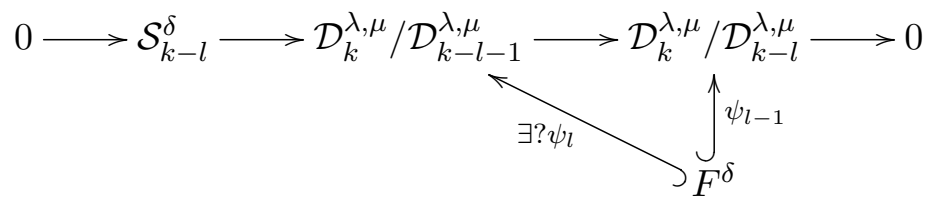

for successively all $l=1, \ldots, k$. With notation as in $(2.4)$, we have $\psi_{l}=\sum_{i=0}^{l} \phi_{i}$. In the next lemma we recover the equivariance condition (2.5) using this cohomological approach.

Lemma 2.4. The partial quantization $\psi_{l}$ defined in (2.7) exists if and only if $\psi_{l-1}$ exists and the 1 -cocycle $\gamma_{l}=\left(\mathcal{L}^{\lambda, \mu}-L^{\delta}\right) \phi_{l-1}$, whose class belongs to $H^{1}\left(\mathfrak{g}, \mathfrak{g}_{-1} \oplus \mathfrak{g}_{0} ; \operatorname{Hom}\left(F^{\delta}, \mathcal{S}_{k-l}^{\delta}\right)\right)$, is trivial. In this case, we have $\psi_{l}=\psi_{l-1}+\phi_{l}$, where $\phi_{l}$ satisfies

$$
\left[\phi_{l}, L_{X}^{\delta}\right]=\gamma_{l}(X)
$$

for some non-zero $X$ in $\mathfrak{g}_{1}$ and $\phi_{l} \in \mathcal{D}_{\mathfrak{g}_{-1} \oplus \mathfrak{g}_{0}}\left(F^{\delta}, \mathcal{S}_{k-l}^{\delta}\right)$.

Proof. Via the normal ordering, $\psi_{l-1}$ lifts linearly to $\mathcal{D}_{k}^{\lambda, \mu} / \mathcal{D}_{k-l-1}^{\lambda, \mu}$. The existence of the morphism $\psi_{l}$ relies then on the triviality of the 1 -cocycle $\left(\mathcal{L}^{\lambda, \mu}-L^{\delta}\right) \psi_{l-1}$. As it takes values in $\mathcal{S}_{k-l}^{\delta}$, and as $\mathcal{L}_{X}^{\lambda, \mu}-L_{X}^{\delta}$ lowers the degree by one, the previous 1 -cocycle is equal to $\gamma_{l}$. Thus $\psi_{l}$ exists if and only if $\gamma_{l}=d \phi_{l}$, and $\psi_{l}=\psi_{l-1}+\phi_{l}$ is the splitting morphism. From the definition of $\gamma_{l}$, we get the $\mathfrak{g}_{-1} \oplus \mathfrak{g}_{0}$-invariance of $\phi_{l}$. Finally, the irreducibility of the action of $\mathfrak{g}_{0}$ on $\mathfrak{g}_{1}$ together with definition of 1-cocycles shows that (2.8) is equivalent to the triviality of $\gamma_{l}$.

\subsection{Main result}

We can now give a characterization of the critical values $\delta$ of the $\mathfrak{g}$-equivariant quantization in terms of $\mathfrak{g}$-invariant operators.

Theorem 2.5. Let $\mathfrak{g}$ be an IFFT-algebra and let $F^{\delta}=\left(F, L^{\delta}\right)$ be a $\mathfrak{g}$-submodule of $\mathcal{S}_{k}^{\delta}$ for every $\delta \in \mathbb{R}$. The $\mathfrak{g}$-equivariant quantization exists and is unique on $F^{\delta}$ if and only if there exists no non-zero $\mathfrak{g}$-invariant differential operator from $F^{\delta}$ to $\mathcal{S}_{k-l}^{\delta}$, for $l=1, \ldots, k$.

Proof. Let $\delta \in I_{k}$, defined in Theorem 2.3. Clearly, if there exists a $\mathfrak{g}$-equivariant quantization on $F^{\delta}$, its uniqueness is equivalent to the absence of $\mathfrak{g}$-invariant operator from $F^{\delta}$ to $\mathcal{S}_{k-l}^{\delta}$ for $l=1, \ldots, k$. We only have to prove that such an absence implies existence of the $\mathfrak{g}$-equivariant quantization on $F^{\delta}$. By induction on $l$, this amounts to obtaining the partial quantization $\psi_{l}$ out of $\psi_{l-1}$. By the preceding lemma, this means to prove that the 1-cocycle $\gamma_{l}^{\delta}$ is trivial: $\gamma_{l}^{\delta}(X) \in\left[E_{0}^{\delta}, L_{X}^{\delta}\right]$ for a non-zero $X$ in $\mathfrak{g}_{1}$ and $E_{0}^{\delta}:=\mathcal{D}_{\mathfrak{g}_{-1} \oplus \mathfrak{g}_{0}}\left(\mathcal{S}_{k}^{\delta}, \mathcal{S}_{k-l}^{\delta}\right)$.

By Theorem 2.3, we know that $\mathfrak{g}$-equivariant quantization exists for shifts $\delta^{\prime} \neq \delta$ in a small enough neighborhood $\mathcal{U}$ of $\delta$. In particular this implies that $\gamma_{l}^{\delta^{\prime}}(X)$ is a coboundary, hence it pertains to the space $\left[E_{0}^{\delta^{\prime}}, L_{X}^{\delta^{\prime}}\right]$. We have to show that this remains true in the limit $\delta^{\prime} \rightarrow \delta$. The Lemma 2.2 ensures that the domains $E_{0}^{\delta^{\prime}}$ are finite-dimensional and independent of $\delta^{\prime}$, so we denote them all by $E_{0}$. We also introduce $E_{1}$, the subspace of $\mathcal{D}\left(\left(\mathcal{S}\left(\mathbb{R}^{n}\right), \mathcal{S}\left(\mathbb{R}^{n}\right)\right)\right.$ generated by the family of spaces $\left[E_{0}, L_{X}^{\delta^{\prime}}\right]$ for $\delta^{\prime} \in \mathbb{R}$. As $X$ is quadratic, we deduce from (2.2) that the space generated by the operators $L_{X}^{\delta^{\prime}}$ is finite-dimensional, and hence $E_{1}$ is also. Consequently, we get a continuous family of linear maps, indexed by $\delta^{\prime} \in \mathbb{R}$, between finite-dimensional spaces:

$$
\left[\cdot, L_{X}^{\delta^{\prime}}\right]: E_{0} \longrightarrow E_{1} .
$$


Since there is no $\mathfrak{g}$-invariant differential operator from $F^{\delta^{\prime}}$ to $\mathcal{S}_{k-l}^{\delta^{\prime}}$ for $\delta^{\prime} \in \mathcal{U}$, the kernel of $\left[\cdot, L_{X}^{\delta^{\prime}}\right]$ is reduced to zero for $\delta^{\prime} \in \mathcal{U}$. Consequently, the spaces $\operatorname{im}\left(\left[\cdot, L_{X}^{\delta^{\prime}}\right]\right)$ are of constant rank on $\mathcal{U}$ and the relation $\gamma_{l}^{\delta^{\prime}}(X) \in \operatorname{im}\left(\left[\cdot, L_{X}^{\delta^{\prime}}\right]\right)$ is preserved in the limit $\delta^{\prime} \rightarrow \delta$.

The latter proof does not give a direct construction of the g-equivariant quantization, but it completes its usual construction in terms of Casimir operators [4]. Indeed, if the latter method fails for a shift $\delta$ whereas the $\mathfrak{g}$-equivariant quantization $\mathcal{Q}^{\lambda, \lambda+\delta}$ exists and is unique, we have just shown that it is recovered from $\mathcal{Q}^{\lambda, \lambda+\delta^{\prime}}$ in the limit $\delta^{\prime} \rightarrow \delta$.

Let $V$ and $W$ be irreducible representations of $\mathfrak{g}_{0}$ of finite dimensions, and let $\mathcal{V}=\mathbb{R}^{n} \times V$, $\mathcal{W}=\mathbb{R}^{n} \times W$ the corresponding trivial bundles over $\mathbb{R}^{n}$. A natural generalization of $\mathfrak{g}$-equivariant quantization is to consider differential operators from $\Gamma(\mathcal{V})$ to $\Gamma(\mathcal{W}) \otimes \mathcal{F}^{\delta}$, the space of symbols being then $\mathcal{S}^{\delta} \otimes \Gamma\left(\mathcal{V}^{*}\right) \otimes \Gamma(\mathcal{W}):=\mathcal{S}^{\delta}(\mathcal{V}, \mathcal{W})$. Theorem 2.3 has been generalized in [6] to this context, and all the results of this section generalize straightforwardly to that situation also (for Lemma 2.2, note that the dilation acts diagonally on $V$ and $W$ ). This leads to the following theorem.

Theorem 2.6. Let $\mathfrak{g}$ be an IFFT-algebra and let $F^{\delta}=\left(F, L^{\delta}\right)$ be a $\mathfrak{g}$-submodule of $\mathcal{S}_{k}^{\delta}(\mathcal{V}, \mathcal{W})$ for any $\delta \in \mathbb{R}$. The $\mathfrak{g}$-equivariant quantization exists and is unique on $F^{\delta}$ if and only if there exists no non-zero $\mathfrak{g}$-invariant differential operator from $F^{\delta}$ to $\mathcal{S}_{k-l}^{\delta}(\mathcal{V}, \mathcal{W})$, for $l=1, \ldots, k$.

Let us mention that we obtain a necessary and sufficient condition for $\delta$ to be a critical value, contrary to the previous works relying on the diagonalization of the Casimir operator on the space of symbols. The sufficient condition obtained there was that specified eigenvalues of this operator are equal. This is clearly a stronger condition on $\delta$ than ours.

\section{Projectively equivariant quantization}

We turn now to the case $\mathfrak{g}=\mathfrak{s l}_{n+1}$ and restrict our consideration to differential operators acting on densities. First, we recall the construction of an explicit formula for the projectively equivariant quantization, using freely results of the original works $[9,17]$. Then we study in detail the critical and resonant values, in particular their link with existence of projectively invariant differential operators. This can be seen as a warm up for the conformal case.

\subsection{Explicit formula}

The projective action of $\mathrm{SL}(n+1, \mathbb{R})$ on the projective space $\mathbb{R} P^{n}$ induces an embedding of $\mathfrak{s l}(n+1, \mathbb{R})$ into the polynomial vector fields on $\mathbb{R}^{n}$. The resulting Lie algebra $\mathfrak{s l}_{n+1}$ of projective vector fields is generated by the affine vector fields and the projective inversions

$$
X^{i}=x^{i} x^{j} \partial_{j}
$$

for $i=1, \ldots, n$. This shows that $\mathfrak{s l}_{n+1}$ is an IFFT-algebra. Recall that for affine vector fields $X$, we have $\mathcal{L}_{X}^{\lambda, \mu}=L_{X}^{\delta}$, so the lack of projective equivariance for the normal ordering is described on $\mathcal{S}_{k}^{\delta}$ by

$$
\mathcal{L}_{X^{i}}^{\lambda, \mu}-L_{X^{i}}^{\delta}=\ell_{k-1}(\lambda) \partial_{p_{i}}
$$

where $\ell_{k}(\lambda)=-(k+\lambda(n+1))$. Resorting to the preceding section, the projectively equivariant quantization on $\mathcal{S}_{k}^{\delta}$ decomposes as $\mathcal{N} \circ\left(\operatorname{Id}+\phi_{1}+\cdots+\phi_{k}\right)$, where $\phi_{m}$ is an $\mathfrak{a f f}(n, \mathbb{R})$-invariant operator lowering the degree by $m$ and satisfying

$$
\left[\phi_{m}, L_{X^{i}}^{\delta}\right]=\gamma_{m}\left(X^{i}\right)
$$


with $\gamma_{m}=\left(\mathcal{L}^{\lambda, \mu}-L^{\delta}\right) \phi_{m-1}$. Weyl's theory of invariants [24], together with Lemma 2.2, shows that the $\mathfrak{a f f}(n, \mathbb{R})$-invariant differential operators acting on symbols are generated by the Euler operator $\mathcal{E}=p_{i} \partial_{p_{i}}$ and the divergence operator $D=\partial_{i} \partial_{p_{i}}$. Hence, restricted to $\mathcal{S}_{k}^{\delta}$, the map $\phi_{m}$ is of the form $c_{m}^{k} D^{m}$, with $c_{m}^{k} \in \mathbb{R}$. This coefficient is determined by substitution into the equation (3.2) and using

$$
\left.\left[D^{m}, L_{X^{i}}^{\delta}\right]\right|_{\mathcal{S}_{k}^{\delta}}=d_{m}^{k}(\delta) \partial_{p_{i}} D^{m-1},
$$

where $d_{m}^{k}(\delta)=m(-2 k+m+1+(\delta-1)(n+1))$.

Theorem 3.1 ([9, 17]). Let $1 \leq l \leq k$. If $\delta \neq 1+\frac{2 k-l-1}{n+1}$, there exists a unique projectively equivariant quantization on $\mathcal{S}_{k}^{\delta}$, given by $\mathcal{N} \circ\left(\sum_{m=0}^{k} c_{m}^{k} D^{m}\right)$, with $c_{0}^{k}=1$ and

$$
c_{m}^{k}=\frac{\ell_{k-m}(\lambda)}{d_{m}^{k}(\delta)} c_{m-1}^{k} .
$$

If $\delta=1+\frac{2 k-l-1}{n+1}$ (a critical value), there exists a projectively equivariant quantization on $\mathcal{S}_{k}^{\delta}$ if and only if $\lambda=\frac{1-h}{n+1}$ with $k-l<h \leq k$ (resonance), and it is given by $\mathcal{N} \circ\left(\sum_{m=0}^{k} c_{m}^{k} D^{m}\right)$, the coefficients $c_{m}^{k}$ being defined as above, except $c_{l}^{k}$ which is free.

\subsection{Critical values and cohomology}

In light of Theorem 2.5, the projectively equivariant quantization exists and is unique on $\mathcal{S}_{k}^{\delta}$ if and only if there is no projectively invariant differential operator acting on this space. From preceding considerations, the only candidates are powers of the divergence $D$, and equation (3.3) shows that $D^{l}$ is projectively invariant on $\mathcal{S}_{k}^{\delta}$ if and only if $\delta=1+\frac{2 k-l-1}{n+1}$. Thus, we recover exactly the statement of Theorem 3.1, and are able to interpret the critical values of $\delta$ in terms of existence of projectively invariant operators on $\mathcal{S}_{k}^{\delta}$. Following Lecomte [16], this can be stated in cohomological terms. Let us introduce the 1-cocycle

$$
\gamma(X)=\frac{1}{n+1} \partial_{i}(\operatorname{Div} X) \partial_{p_{i}} D^{l-1}
$$

whose class lies in $H^{1}\left(\mathfrak{s l}_{n+1}, \operatorname{Hom}\left(\mathcal{S}_{k}^{\delta}, \mathcal{S}_{k-l}^{\delta}\right)\right)$. It satisfies the equality $\gamma_{l}=\left(\ell_{k-l}(\lambda) c_{k-l+1}^{k}\right) \gamma$. Since $\gamma$ vanishes on $\mathfrak{a f f}(n, \mathbb{R})$ and $\operatorname{Hom}_{\mathfrak{a f f}}\left(\mathcal{S}_{k}^{\delta}, \mathcal{S}_{k-l}^{\delta}\right)$ is generated by $D^{l}$, we deduce from (3.3) that $\gamma$ defines a nontrivial 1-cocycle if and only if $D^{l}$ is projectively invariant, i.e. $\delta=1+\frac{2 k-l-1}{n+1}$. Consequently, for this critical value of $\delta$, we get an obstruction to the existence of a projectively equivariant quantization except when $\gamma_{l}$ vanishes. This occurs when

$$
\forall X \in \mathfrak{s l}_{n+1},\left.\quad\left(\mathcal{L}_{X}^{\lambda, \mu}-L_{X}^{\delta}\right)\right|_{\mathcal{S}_{h}^{\delta}}=0,
$$

or equivalently when $\ell_{h-1}(\lambda)=0$ for some $k-l<h \leq k$, giving the resonant values $(\lambda, \lambda+\delta)$.

\subsection{Resonances and projectively invariant operators}

Here we provide an interpretation of the resonant values of $\lambda$ in terms of projectively invariant operators acting on the space of densities $\mathcal{F}^{\lambda}$.

Theorem 3.2. The projectively equivariant quantization $\mathcal{Q}^{\lambda, \mu}: \mathcal{S}_{k}^{\delta} \otimes \mathcal{F}^{\lambda} \rightarrow \mathcal{F}^{\mu}$ exists and is unique except when there is a non-zero projectively invariant differential operator: $\mathcal{S}_{k}^{\delta} \rightarrow \mathcal{S}_{k-l}^{\delta}$. In that case, $\mathcal{Q}^{\lambda, \mu}$ exists if and only if there is a projectively invariant differential operator of order $h$ from $\mathcal{F}^{\lambda}$ to sections of a homogeneous bundle, with $k-l<h \leq k$. 
Proof. The first statement has just been proven. The second one relies on the characterization by equation (3.4) of the resonant values $(\lambda, \lambda+\delta)$. Indeed, this relation provides a way to generate projectively invariant operators.

Lemma 3.3. Let $\left.\left(\mathcal{L}_{X}^{\lambda, \mu}-L_{X}^{\delta}\right)\right|_{\mathcal{S}_{k}^{\delta}}=0$ for all $X \in \mathfrak{g}$, a Lie subalgebra of $\operatorname{Vect}\left(\mathbb{R}^{n}\right)$, and let $\mathcal{B}$ be the space of sections of a homogeneous bundle. If there exists a $\mathfrak{g}$-invariant element in $\mathcal{S}_{k}^{\delta} \otimes \mathcal{B}$, then its image by normal ordering is a $\mathfrak{g}$-invariant differential operator: $\mathcal{F}^{\lambda} \rightarrow \mathcal{F}^{\mu} \otimes \mathcal{B}$ of order $k$.

Proof. The expression $\mathcal{L}_{X}^{\lambda, \mu}-L_{X}^{\delta}$ is the same if, on the one hand, $L^{\delta}$ is the action on $\mathcal{S}_{k}^{\delta}$ and $\mathcal{L}^{\lambda, \mu}$ is the one on $\mathcal{D}_{k}^{\lambda, \mu}$, and if, on the other hand, $L^{\delta}$ is the action on $\mathcal{S}_{k}^{\delta} \otimes \mathcal{B}$ and $\mathcal{L}^{\lambda, \mu}$ is the action on the differential operators from $\mathcal{F}^{\lambda}$ to $\mathcal{F}^{\mu} \otimes \mathcal{B}$ of order $k$.

Consequently, we want to obtain $\mathfrak{s l}_{n+1}$-invariant elements. Using Weyl's theory of invariants of $\operatorname{gl}_{n}(\mathbb{R})\left(\subset \mathfrak{s l}_{n+1}\right)$, we have no choice but to take $\mathcal{B}=\overline{\mathcal{S}}^{-\delta}=\left(\overline{\mathcal{S}}\left(\mathbb{R}^{n}\right), \bar{L}^{-\delta}\right)$, the module of functions on $T M$ polynomial in the fiber variables tensored with $(-\delta)$-densities. Denoting the fiber coordinates by $\bar{p}^{i}$, the $\mathrm{gl}_{n}(\mathbb{R})$-invariant elements are $\left(\bar{p}^{i} p_{i}\right)^{h} \in \mathcal{S}_{h}^{\delta} \otimes \overline{\mathcal{S}}_{h}^{-\delta}$ for $k \in \mathbb{N}$. They are obviously $\mathfrak{s l}_{n+1}$-invariant, and by Lemma 3.3 and equation (3.1), they gives rise for $\ell_{h-1}(\lambda)=0$ to a projectively invariant differential operator

$$
\bar{G}^{h}: \mathcal{F}^{\lambda} \rightarrow \overline{\mathcal{S}}_{h}^{\lambda}
$$

with $\bar{G}=\bar{p}^{i} \partial_{i}$. As shown by straightforward computations, this is the only projectively invariant differential operator with principal symbol $\left(\bar{p}^{i} p_{i}\right)^{h}$. Thus, there is a projectively invariant differential operator of order $h$ on $\mathcal{F}^{\lambda}$ if and only if $\lambda=\frac{1-h}{n+1}$, and the theorem is proved.

Now we make concrete the existence of projectively equivariant quantization for resonances, by constructing it from the projectively invariant operators (3.5). Let us denote by $\mathcal{D}\left(\overline{\mathcal{S}}_{k}^{\lambda}, \mathcal{F}^{\mu}\right)$ the space of differential operators from $\overline{\mathcal{S}}_{k}^{\lambda}$ to $\mathcal{F}^{\mu}$, which is isomorphic to $\mathcal{D}^{\lambda, \mu} \otimes \mathcal{S}_{k}^{0}$ as $\operatorname{Vect}\left(\mathbb{R}^{n}\right)$ module. The $\operatorname{Vect}\left(\mathbb{R}^{n}\right)$-invariant 1 -form $\alpha=d x^{i} \partial_{\bar{p}^{i}}$ acts by interior product on $\operatorname{Vect}\left(\mathbb{R}^{n}\right)$ and vanishes on $\mathcal{S}_{k}^{\delta}$. Thus, it extends as a derivation, denoted by $\iota_{\alpha}$, on the algebra underlying $\mathcal{D}^{\lambda, \mu} \otimes \mathcal{S}^{0}$ and gives rise for any integer $j \leq k$ to the following morphism of $\operatorname{Vect}\left(\mathbb{R}^{n}\right)$-modules,

$$
\left(\iota_{\alpha}\right)^{j}: \mathcal{D}_{k}^{\lambda, \mu} \rightarrow \mathcal{D}_{k-j}\left(\overline{\mathcal{S}}_{j}^{\lambda}, \mathcal{F}^{\mu}\right),
$$

with kernel $\mathcal{D}_{j-1}^{\lambda, \mu}$. From projective invariance of the operators given in (3.5), we deduce the following proposition.

Proposition 3.4. Let $\delta=1+\frac{2 k-l-1}{n+1}$ and $\lambda=\frac{1-h}{n+1}$, where $k-l<h \leq k$. Then, the partial projectively equivariant quantization $\mathcal{Q}_{k, h}^{\lambda, \mu}=\mathcal{N} \circ\left(\mathrm{Id}+\cdots+\phi_{h-1}\right)$ exists and induces the following commutative diagram of $\mathfrak{s l}_{n+1}$-modules,

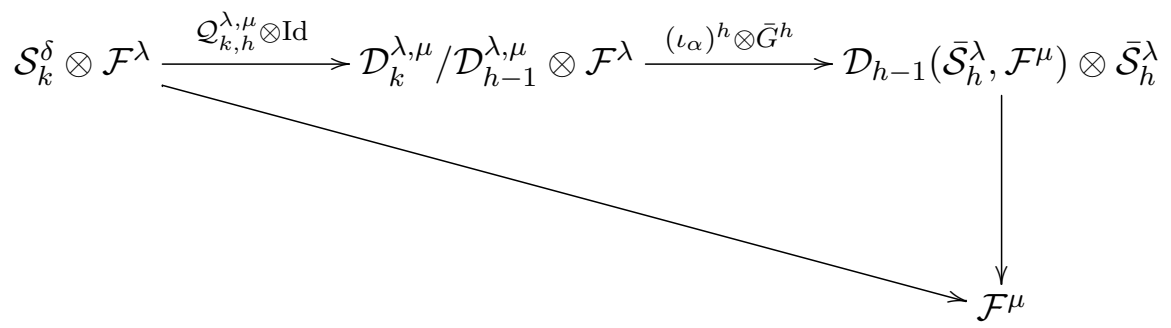

This defines a projectively equivariant quantization on $\mathcal{S}_{k}^{\delta}$. 


\section{Conformally equivariant quantization and invariant operators}

The aim of this section is to obtain a full characterization of existence of the conformally equivariant quantization in the spirit of Theorem 3.2. The task is harder in this case since the spaces $D_{\mathfrak{g}_{-1} \oplus \mathfrak{g}_{0}}\left(\mathcal{S}_{k, s}^{\delta}, \mathcal{S}_{l, t}^{\delta}\right)$ are generically multi-dimensional (see (4.4) for notation $\mathcal{S}_{*, *}^{\delta}$ ).

\subsection{The conformal Lie algebra}

Let $\left(x^{i}\right)$ denote the cartesian coordinates on $\mathbb{R}^{n}$ and $\eta$ be the canonical flat metric of signature $(p, q)$. The Lie algebra of conformal Killing vector fields on $\left(\mathbb{R}^{n}, \eta\right)$, denoted by $\mathfrak{c} f$ for short, is isomorphic to $\mathfrak{o}(p+1, q+1)$. As a subalgebra of polynomial vector fields it admits a gradation $\mathfrak{c} \mathfrak{f}=\mathfrak{c f}_{-1} \oplus \mathfrak{c f}_{0} \oplus \mathfrak{c f}_{1}$ by the degree of the vector field components, this is a particular case of IFFT-algebra. The Lie subalgebra $\mathfrak{c f}_{-1}$ consists of the translations and $\mathfrak{c f}_{0}$ consists of the linear conformal transformations. Thus, $\mathfrak{c f}$ is generated by their sum $\mathfrak{c} e=\mathfrak{c} \mathfrak{f}-1 \oplus \mathfrak{c f}_{0}$, and by the following conformal inversions in $\mathfrak{c f}_{1}$,

$$
X_{i}=x_{j} x^{j} \partial_{i}-2 x_{i} x^{j} \partial_{j}
$$

where $x_{i}=\eta_{i j} x^{j}$ and $i=1, \ldots, n$. As $\mathfrak{c} \subset \mathfrak{a f f}(n, \mathbb{R})$, we have $\mathcal{L}_{X}^{\lambda, \mu}=L_{X}^{\delta}$ for every $X \in \mathfrak{c} \mathfrak{c}$, and the lack of conformal equivariance for the normal ordering is described by, see [7],

$$
\mathcal{L}_{X_{i}}^{\lambda, \mu}-L_{X_{i}}^{\delta}=-p_{i} T+2(\mathcal{E}+n \lambda) \partial_{p^{i}},
$$

where $X_{i}$ is the conformal inversion (4.1) and $T=\eta_{i j} \partial_{p_{i}} \partial_{p_{j}}, \mathcal{E}=p_{i} \partial_{p_{i}}$.

\subsection{Similarity invariant differential operators}

The first step is to describe the space of $\mathfrak{c}$-invariant operators $\mathcal{D}_{\mathfrak{c e}}\left(\mathcal{S}_{k}^{\delta}, \mathcal{S}_{k^{\prime}}^{\delta}\right)$ for arbitrary $k$ and $k^{\prime}$. Weyl's theory of invariants [24] insures that the algebra of isometry-invariant differential operators acting on $\mathcal{S}^{\delta}$ is generated by

$$
R=\eta^{i j} p_{i} p_{j}, \quad \mathcal{E}=p_{i} \partial_{p_{i}}, \quad T=\eta_{i j} \partial_{p_{i}} \partial_{p_{j}},
$$

corresponding respectively to the metric (or kinetic energy), the Euler operator, the trace operator, and by

$$
G=\eta^{i j} p_{i} \partial_{j}, \quad D=\partial_{i} \partial_{p_{i}}, \quad L=\eta^{i j} \partial_{i} \partial_{j},
$$

corresponding respectively to the gradient, the divergence and the Laplacian. They all become $\mathfrak{c} \mathfrak{c}$-invariant operators if we consider them as operators from $\mathcal{S}^{\delta}$ to $\mathcal{S}^{\delta^{\prime}}$ with a certain shift of weight $\delta^{\prime}-\delta$ given in the following table,

\begin{tabular}{|c|c|c|c|}
\hline values of $n\left(\delta^{\prime}-\delta\right)$ & -2 & 0 & 2 \\
\hline $\mathfrak{c} \mathcal{e}$-invariant operators & $T$ & $\mathcal{E}, D$ & $R, G, L$ \\
\hline
\end{tabular}

The computation of the action of $X_{i} \in \mathfrak{c f}_{1}$ on those operators proves that $\mathcal{E}, R$ and $T$ are conformally invariant, and the table (4.3) ensures that $\mathcal{E}$ and $R T$ preserve the shift. The joint eigenspaces of these two operators define a decomposition of $\mathcal{S}^{\delta}$ into $\mathfrak{c f}$-submodules, which corresponds to the spherical harmonic decomposition in the $p$ variables,

$$
\mathcal{S}^{\delta}=\bigoplus_{k, s \in \mathbb{N}, 2 s \leq k} \mathcal{S}_{k, s}^{\delta}
$$


More precisely, $\mathcal{S}_{k, s}^{\delta}$ is the space of homogeneous symbols of degree $k$ of the form $P=R^{s} Q$ with $T Q=0$. Let $2 s^{\prime} \leq k^{\prime}$ be two integers. Then, each $\mathfrak{c} \mathfrak{e}$ - or $\mathfrak{c f}$-invariant operator $\mathcal{S}_{k, s}^{\delta} \rightarrow \mathcal{S}_{k^{\prime}, s^{\prime}}^{\delta}$ gives rise to the following commutative diagram of $\mathfrak{c} \mathfrak{e}$ - or $\mathfrak{c} \mathfrak{f}$-modules,

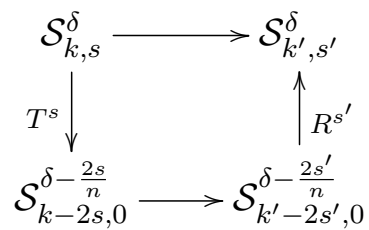

We write $G_{0}, D_{0}, L_{0}$ for the restriction and corestriction of the operators $G, D, L$ to ker $T$. Using the relation $[D, G]=L$, we obtain from (4.3) and (4.5) that $\mathcal{D}_{\mathfrak{c e}}\left(\mathcal{S}_{k, s}^{\delta}, \mathcal{S}_{k^{\prime}, s^{\prime}}^{\delta}\right)$ is linearly generated by the monomials $R^{s^{\prime}} G_{0}^{g} L_{0}^{\ell} D_{0}^{d} T^{s}$, such that $g+\ell=s-s^{\prime}$ and $d+g+2 \ell=k-k^{\prime}$. An explicit description for possibly different weights and $s^{\prime}=s=0$ follows.

Proposition 4.1. Let $k, k^{\prime}$ be integers, $\delta, \delta^{\prime} \in \mathbb{R}$, and define $\ell=\frac{n}{2}\left(\delta^{\prime}-\delta\right)-\max \left(k^{\prime}-k, 0\right)$. The space $\mathcal{D}_{\mathfrak{c e}}\left(\mathcal{S}_{k, 0}^{\delta}, \mathcal{S}_{k^{\prime}, 0}^{\delta^{\prime}}\right)$ is nontrivial only if $\ell$ is a non-negative integer, and then

$$
\mathcal{D}_{\mathfrak{c e}}\left(\mathcal{S}_{k, 0}^{\delta}, \mathcal{S}_{k^{\prime}, 0}^{\delta^{\prime}}\right)= \begin{cases}\left(G_{0}\right)^{k^{\prime}-k}\left\langle L_{0}^{\ell}, G_{0} L_{0}^{\ell-1} D_{0}, \ldots, G_{0}^{\ell} D_{0}^{\ell}\right\rangle & \text { for } k^{\prime}-k \geq 0, \\ \left\langle L_{0}^{\ell}, G_{0} L_{0}^{\ell-1} D_{0}, \ldots, G_{0}^{\ell} D_{0}^{\ell}\right\rangle\left(D_{0}\right)^{k-k^{\prime}} & \text { otherwise. }\end{cases}
$$

From this we get a diagrammatic representation of the spaces $\mathcal{D}_{\mathfrak{c} e}\left(\mathcal{S}_{k, 0}^{\delta}, \mathcal{S}_{k^{\prime}, 0}^{\delta^{\prime}}\right)$, one path between two spaces corresponding to a one-dimensional subspace of $\mathfrak{c}$-invariant operators,

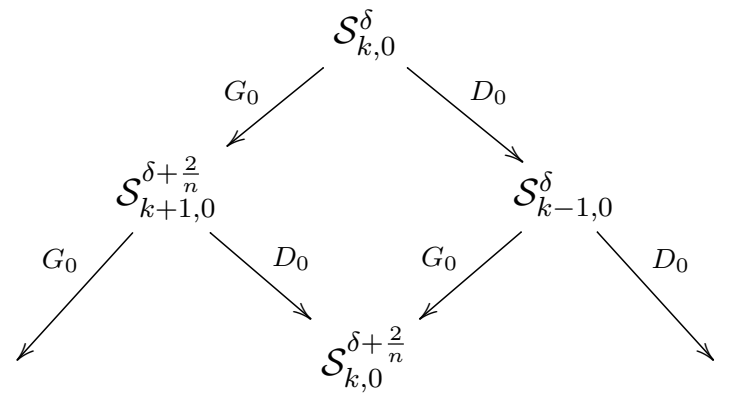

Since $\left[D_{0}, G_{0}\right]=L_{0}$, two paths give rise to independent subspaces if they reach different furthest right column.

\subsection{Classification of conformally invariant differential operators acting on symbols}

Resorting to the decomposition (4.4) of $\mathcal{S}^{\delta}$ into $\mathfrak{c} \mathfrak{f}$-submodules, the classification of conformally invariant differential operators on $\mathcal{S}^{\delta}$ amounts to the determination of the $\mathfrak{c} f$-invariant differential operators between submodules $\mathcal{S}_{k, s}^{\delta}$ and $\mathcal{S}_{k^{\prime}, s^{\prime}}^{\delta}$. The commutative diagram (4.5) of $\mathfrak{c f}$-modules further reduces the quest to the space $\mathcal{D}_{\mathfrak{c f}}\left(\mathcal{S}_{k, 0}^{\delta}, \mathcal{S}_{k^{\prime}, 0}^{\delta^{\prime}}\right)$. The two involved submodules of symbols are modules of sections of irreducible homogeneous bundles associated to the principal fiber bundle over $\left(\mathbb{R}^{n}, \eta\right)$ of conformal linear frames. Consequently, conformally invariant operators correspond to morphisms of generalized Verma modules. Their classification has been performed by Boe and Collingwood [2, 3], see also [10] for a clear summary. This has been translated as a classification of conformally invariant differential operators by Eastwood and Rice for $n=4$ [11]. Instead applying such an heavy machinery to our peculiar case, we prefer to proceed in a direct and elementary way. 
We compute the space $\mathcal{D}_{\mathfrak{c f}}\left(\mathcal{S}_{k, 0}^{\delta}, \mathcal{S}_{k^{\prime}, 0}^{\delta^{\prime}}\right)$ of conformally invariant operators by searching the elements of $\mathcal{D}_{\mathfrak{c e}}\left(\mathcal{S}_{k, 0}^{\delta}, \mathcal{S}_{k^{\prime}, 0}^{\delta^{\prime}}\right)$ which are invariant under the action of a certain $X \in \mathfrak{c f}_{1}$. Concerning the $\mathfrak{c}$-invariant operators $D_{0}^{d}, G_{0}^{g}$ and $L_{0}^{\ell}$, with source space $\mathcal{S}_{k, 0}^{\delta}$, the action of the conformal inversion $X_{i}$ follows from [7],

$$
\begin{aligned}
& {\left[D_{0}^{d}, L_{X_{i}}^{\delta}\right]=2 d(2 k-d-1+n(1-\delta)) \partial_{p^{i}} D_{0}^{d-1},} \\
& {\left[G_{0}^{g}, L_{X_{i}}^{*}\right]=-2 g(n \delta+g-1) \pi_{0} p_{i} G_{0}^{g-1},} \\
& {\left[L_{0}^{\ell}, L_{X_{i}}^{*}\right]=2 \ell\left[(2(k-\ell)+n(1-2 \delta)) \partial_{i}+2\left(G_{0} \partial_{p^{i}}-\pi_{0} p_{i} D_{0}\right)\right] L_{0}^{\ell-1},}
\end{aligned}
$$

where $L_{X_{i}}^{*}$ is defined in (2.3) and $\pi_{0}$ is the conformally invariant projection $\pi_{0}: \mathcal{S}^{\delta} \rightarrow \operatorname{ker} T$. Since the latter operators generate the spaces $\mathcal{D}_{\mathfrak{c} e}\left(\mathcal{S}_{k, 0}^{\delta}, \mathcal{S}_{k^{\prime}, 0}^{\delta^{\prime}}\right)$ with arbitrary $k^{\prime}, \delta^{\prime}$, we deduce that,

$$
\left[\mathcal{D}_{\mathfrak{c e}}\left(\mathcal{S}_{k, 0}^{\delta}, \mathcal{S}_{k^{\prime}, 0}^{\delta^{\prime}}\right), L_{X_{i}}^{*}\right] \subset E_{D} \partial_{p^{i}} \oplus \pi_{0} p_{i} E_{G} \oplus \partial_{i} E_{L},
$$

with $E_{G}, E_{D}, E_{L}$ the maximal vector spaces generated by the three operators $G_{0}, D_{0}$, $L_{0}$, and such that $G_{0} E_{G}, E_{D} D_{0}, L_{0} E_{L} \subset \mathcal{D}_{\mathfrak{c e}}\left(\mathcal{S}_{k, 0}^{\delta}, \mathcal{S}_{k^{\prime}, 0}^{\delta^{\prime}}\right)$. The independence of the monomials $G_{0}^{g} D_{0}^{d} L_{0}^{\ell}$ for different exponents $\ell$ and the commutative diagram (4.5) lead to the next two results.

Lemma 4.2. Let $k, k^{\prime}$ be non-negative integers, $\delta, \delta^{\prime} \in \mathbb{R}$, and let us define $j=\frac{n}{2}\left(\delta^{\prime}-\delta\right)$. The space of conformally invariant operators $\mathcal{D}_{\mathfrak{c f}}\left(\mathcal{S}_{k, 0}^{\delta}, \mathcal{S}_{k^{\prime}, 0}^{\delta^{\prime}}\right)$ is either trivial or of dimension 1 , generated by

- $D_{0}^{d}$ if $k-k^{\prime}=d, j=0$ and $\delta=1+\frac{2 k-d-1}{n}$,

- $G_{0}^{g}$ if $k^{\prime}-k=g, j=g$ and $\delta=\frac{1-g}{n}$,

- $\mathcal{L}_{\ell}$ if $k^{\prime}=k, j=\ell$ and $\delta=\frac{1}{2}+\frac{k-\ell}{n}$,

where the operator $\mathcal{L}_{\ell}$ is of the form $L_{0}^{\ell}+a_{1} G_{0} L_{0}^{\ell-1} D_{0}+\cdots+a_{\ell} G_{0}^{\ell} D_{0}^{\ell}$ for $a_{i} \in \mathbb{R}$. Moreover, the operator $\mathcal{L}_{\ell}$ is not the $\ell^{\text {th }}$ power of $\mathcal{L}_{1}$.

Proof. Let $A \in \mathcal{D}_{\mathfrak{c f}}\left(\mathcal{S}_{k, 0}^{\delta}, \mathcal{S}_{k^{\prime}, 0}^{\delta^{\prime}}\right)$. Resorting to Proposition 4.1, up to a constant we have $A=$ $G_{0}^{g} B D_{0}^{d}$ where $B=L_{0}^{\ell}+a_{1} G_{0} L_{0}^{\ell-1} D_{0}+\cdots+a_{\ell} G_{0}^{\ell} D_{0}^{\ell}$, for some integers $g, d, \ell$ and reals $a_{1}, \ldots, a_{\ell}$. As the component in $E_{D} \partial_{p^{i}}$ of the higher degree term in $L_{0}$ of $\left[A, L_{X_{i}}^{*}\right]$ vanishes, we get

$$
\left[D_{0}^{d}, L_{X_{i}}^{*}\right]=0 .
$$

From the relation (4.7) we deduce that necessarily $\delta=1+\frac{2 k-d-1}{n}$ if $d \neq 0$. As the component in $\pi_{0} p_{i} E_{G}$ of the higher degree term in $L_{0}$ of $\left[A, L_{X_{i}}^{*}\right]$ vanishes, we obtain

$$
\left[G_{0}^{g}, L_{X_{i}}^{*}\right]=0 .
$$

From the relation (4.8) we deduce that necessarily $\delta+\frac{2 \ell}{n}=\frac{1-g}{n}$ if $g \neq 0$. As a consequence $B$ must be conformally invariant, and since the component in $\partial_{i} E_{L}$ of the higher degree term in $L_{0}$ of $\left[B, L_{X_{i}}^{*}\right]$ vanishes, we are lead to

$$
\left[L_{0}^{\ell}, L_{X_{i}}^{*}\right] \in \pi_{0} p_{i} E_{G} \oplus E_{D} \partial_{p^{i}} .
$$

From the relation (4.9) we deduce that necessarily $\delta=\frac{1}{2}+\frac{k-\ell}{n}$ if $\ell \neq 0$. Then, straightforward but lengthy computations show that there exist unique reals $a_{1}, \ldots, a_{\ell}$ such that $L_{0}^{\ell}+a_{1} G_{0} L_{0}^{\ell-1} D_{0}+$ $\cdots+a_{\ell} G_{0}^{\ell} D_{0}^{\ell}$ is conformally invariant, and the expression of $a_{1}$ ensures that $\mathcal{L}_{\ell} \neq\left(\mathcal{L}_{1}\right)^{\ell}$. The three values found for $\delta$ are incompatible two by two, hence we get that among the exponents $g, d, \ell$ one at most is non-vanishing. The result follows. 
Theorem 4.3. Let $k \geq 2 s$ and $k^{\prime} \geq 2 s^{\prime}$ be integers, and $\delta, \delta^{\prime} \in \mathbb{R}$. The space of conformally invariant differential operators $\mathcal{D}_{\mathfrak{c f}}\left(\mathcal{S}_{k, s}^{\delta}, \mathcal{S}_{k^{\prime}, s^{\prime}}^{\delta^{\prime}}\right)$ is either trivial or of dimension 1 . In the latter case $j=\frac{n}{2}\left(\delta^{\prime}-\delta\right)$ is an integer and the space is generated by

- $R^{s^{\prime}} D^{d} T^{s}$, if $s^{\prime}-s=j, k-k^{\prime}=d-2 j$ and $\delta=1+\frac{2(k-s)-d-1}{n}$,

- $R^{s^{\prime}} G_{0}^{g} T^{s}$, if $g+s^{\prime}-s=j, k-k^{\prime}=s-s^{\prime}-j$ and $\delta=\frac{2 s+1-g}{n}$,

- $R^{s^{\prime}} \mathcal{L}_{\ell} T^{s}$, if $\ell+s^{\prime}-s=j, k-k^{\prime}=2(\ell-j)$ and $\delta=\frac{1}{2}+\frac{k-\ell}{n}$.

Remark 4.4. If $n=2,3$, there is also isometric invariants in $\mathcal{D}(\mathcal{S}, \mathcal{S})$ built from the volume form, but the only conformally invariant one is the algebraic operator $p_{1} \partial_{p_{2}}-p_{2} \partial_{p_{1}}$ for $n=2$.

Remark 4.5. The conformally invariant operator $G_{0}^{g}$ is the $g$-generalized conformal Killing operator, whose kernel is the space of $g$-generalized conformal Killing tensors [21]. The conformally invariant operator $\mathcal{L}_{\ell}$ is the generalization of $\ell^{\text {th }}$ power of the Laplacian to trace free symbols, its curved analog, for $\ell=1$, has been obtained in [25].

\subsection{Critical values and cohomology}

From Theorem 4.3, classifying the conformally invariant differential operators on $\mathcal{S}^{\delta}$, and Theorem 2.5, characterizing the critical values of the shift $\delta$ in terms of invariant differential operators, we recover the following theorem of Silhan.

Theorem 4.6 ([23]). Let $k \geq 2 s$ and $l$ be three integers and $\delta \in \mathbb{R}$. The conformally equivariant quantization exists and is unique on $\mathcal{S}_{k, s}^{\delta}$ if and only if there is no conformally invariant differential operator $\mathcal{S}_{k, s}^{\delta} \rightarrow \mathcal{S}_{k-l}^{\delta}$. This means $\delta \notin I_{k, s}$, where the set of critical values is of the following form: $I_{k, s}=I_{k, s}^{D} \amalg\left(I_{k, s}^{G} \cup I_{k, s}^{L}\right)$ and

$$
\begin{aligned}
& I_{k, s}^{D}=\left\{1+\frac{2(k-s)-d-1}{n} \mid d \in \llbracket 1, k-2 s \rrbracket\right\}, \\
& I_{k, s}^{G}=\left\{\frac{2 s+1-g}{n} \mid g \in \llbracket 1, s \rrbracket\right\}, \quad I_{k, s}^{L}=\left\{\frac{1}{2}+\frac{k-\ell}{n} \mid \ell \in \llbracket 1, s \rrbracket\right\} .
\end{aligned}
$$

We provide now an alternative characterization of the critical values $\delta \in I_{k, s}$ in cohomological terms. The Proposition 4.1 and the commutative diagram (4.5) allow to decompose the conformally equivariant quantization $\mathcal{N} \circ Q^{\lambda, \mu}$ on $\mathcal{S}_{k, s}^{\delta}$ as

$$
Q^{\lambda, \mu}=\sum_{g \leq s}\left(\sum_{d \leq k-2 s+g} \phi_{d, g}\right),
$$

with $\phi_{d, g} \in \mathcal{D}_{\mathfrak{c e}}\left(\mathcal{S}_{k, s}^{\delta}, \mathcal{S}_{k-d-g, s-g}^{\delta}\right)$ by $\mathfrak{c}$-invariance of the normal ordering. Analogously to (4.10), we introduce the spaces $E_{d, g}=R^{s^{\prime}}\left(E_{D} \partial_{p^{i}} \oplus \pi_{0} p_{i} E_{G} \oplus \partial_{i} E_{L}\right) T^{s}$, which are the sum of three maximal subspaces of invariant operators such that $R^{s^{\prime}} E_{D} D_{0} T^{s}, \ldots \subset \mathcal{D}_{\mathfrak{c e}}\left(\mathcal{S}_{k, s}^{\delta}, \mathcal{S}_{k-d-g, s-g}^{\delta}\right)$. From Proposition 4.1, we easily get that $\operatorname{dim} E_{d, 0}=\operatorname{dim} E_{0, g}=1$, and denoting $\min (d, g)$ by $\ell$, we also obtain that $\operatorname{dim} E_{d, g}=3 \ell+1$ if $g \neq d$ and $\operatorname{dim} E_{\ell, \ell}=3 \ell$.

Lemma 4.7. Let $X_{i} \in \mathfrak{c f}_{1}$. The map $\gamma \mapsto \gamma\left(X_{i}\right)$ establishes a linear isomorphism between $E_{d, g}$ and the space of 1-cocycles vanishing on $\mathfrak{c}$ and with values in $\operatorname{Hom}\left(\mathcal{S}_{k, s}^{\delta}, \mathcal{S}_{k-d-g, s-g}^{\delta}\right)$.

Proof. Let $X_{i}$ and $X_{j}$ be inversions given by (4.1). From the cocycle relation (2.6) and the vanishing of $\gamma$ on $\mathfrak{c}$, we get that $\eta^{i j} \gamma\left(X_{j}\right) \partial_{i}$ is $\mathfrak{c} \mathfrak{e}$-invariant. This proves that the image of the considered map lies in $E_{d, g}$. Let $A \in E_{d, g}$ and $X_{j i}=x_{j} \partial_{i}-x_{i} \partial_{j} \in \mathfrak{c f}_{0}$. The latter satisfies $\left[X_{j i}, X_{i}\right]=X_{j}$, and allows to define a linear map on $\mathfrak{c} \mathfrak{f}$ by: $\gamma(X)=0$ if $X \in \mathfrak{c} e, \gamma\left(X_{i}\right)=A$ and $\gamma\left(X_{j}\right)=\left[L_{X_{j i}}^{\delta}, A\right]$ if $j \neq i$. This map is a 1-cocycle satisfying the required properties. 
Proposition 4.8. The relative cohomology space $\mathrm{H}^{1}\left(\mathfrak{c} \mathfrak{f}, \mathfrak{c} ; \operatorname{Hom}\left(\mathcal{S}_{k, s}^{\delta}, \mathcal{S}^{\delta}\right)\right)$ changes of dimension exactly for the critical values $\delta \in I_{k, s}$. Then it rises by one, or two if $\delta \in I_{k, s}^{G} \cap I_{k, s}^{L}$. In particular, the 1-cocycles

$$
\gamma_{d}: X \mapsto \partial_{i}(\operatorname{Div} X) \partial_{p_{i}} D^{d-1}, \quad \gamma_{g}: X \mapsto \eta^{i j} \partial_{i}(\operatorname{Div} X) R^{s-g} \pi_{0} p_{j} G_{0}^{g-1} T^{s}
$$

are nontrivial if and only if the operators $D^{d}$ and $R^{s-g} G_{0}^{g} T^{s}$ are $\mathfrak{c} \mathfrak{f}$-invariant.

Proof. According to the proof of the previous Lemma, if $[\gamma] \in \mathrm{H}^{1}\left(\mathfrak{c} \mathfrak{f}, \mathfrak{c} \mathfrak{c} ; \operatorname{Hom}\left(\mathcal{S}_{k, s}^{\delta}, \mathcal{S}^{\delta}\right)\right)$, then $\eta^{i j} \partial_{i} \gamma\left(X_{j}\right)$ is $\mathfrak{c} \mathfrak{e}$-invariant. Consequently, the space $\mathrm{H}^{1}\left(\mathfrak{c} \mathfrak{f}, \mathfrak{c} \mathfrak{c} ; \operatorname{Hom}\left(\mathcal{S}_{k, s}^{\delta}, \mathcal{S}^{\delta}\right)\right)$ is the direct sum of the spaces $\mathrm{H}^{1}\left(\mathfrak{c} \mathfrak{f}, \mathfrak{c} \mathfrak{i} ; \operatorname{Hom}\left(\mathcal{S}_{k, s}^{\delta}, \mathcal{S}_{k-d-g, s-g}^{\delta}\right)\right)$ for $g \leq s$ and $d \leq k-2 s+g$. The spaces of corresponding 1-cocycles have just been identified to the spaces $E_{d, g}$, whose dimension is known and independent of $\delta$. The spaces of corresponding 1-coboundaries have a dimension equal to that of $\mathcal{D}_{\mathfrak{c e}}\left(\mathcal{S}_{k, s}^{\delta}, \mathcal{S}_{k-d-g, s-q}^{\delta}\right)$ minus the one of the subspaces of $\mathfrak{c} f$-invariant elements. The latter is zero generically, except for the critical values $\delta \in I_{k, s}$, hence the result.

For generic $\delta$, the two given maps $\gamma_{d}$ and $\gamma_{g}$ are 1-coboundaries, proportional to the differential of the operators $D^{d}$ and, respectively, $R^{s-g} G_{0}^{g} T^{s}$. Consequently, they define 1-cocycles for every $\delta \in \mathbb{R}$. Since they vanish on $\mathfrak{c}$, they are trivial only if they are of the form $X \mapsto\left[A, L_{X}^{\delta}\right]$, with $A \in \mathcal{D}_{\mathfrak{c} \mathfrak{e}}\left(\mathcal{S}_{k, s}^{\delta}, \mathcal{S}_{k^{\prime}, s^{\prime}}^{\delta}\right)$ for the adapted values of $k^{\prime}$ and $s^{\prime}$. This space reduces to 0 if $D^{d}$ and $R^{s-g} G_{0}^{g} T^{s}$ are $\mathfrak{c} f$-invariant, leading to the announced result.

\subsection{Resonances and conformally invariant differential operators}

The pairs of weights $(\lambda, \mu)$ for which the conformally equivariant quantization exists are completely known only on symbols of degrees 2 and 3 in the momenta $[8,18]$. Such a pair is called a resonance and in the known cases there are a finite number of resonances for a given critical value of the shift $\delta=\mu-\lambda$. We extend those results to the general case and provide a complete classification for existence of the conformally equivariant quantization.

Theorem 4.9. Let $k \geq 2 s$ be two integers and $\delta \in I_{k, s}$. Restricted to the submodule $\mathcal{S}_{k, s}^{\delta}$, the existence of the conformally equivariant quantization is equivalent to

$$
\begin{array}{lll}
\text { (i) } \lambda=\frac{1-h}{n}, k-s-d<h \leq k-s & \text { if } \delta=1+\frac{2(k-s)-d-1}{n} \in I_{k, s}^{D}, \\
\text { (ii) } \lambda=\frac{n-2 t}{2 n}, s-g<t \leq s & \text { if } \delta=\frac{2 s+1-g}{n} \in I_{k, s}^{G} \backslash I_{k, s}^{L}, \\
\text { (iii) } \lambda=\frac{1-h}{n}, k-s-\ell<h \leq k-s & \text { if } \delta=\frac{1}{2}+\frac{k-\ell}{n} \in I_{k, s}^{L} \backslash I_{k, s}^{G}, \\
\text { (iii) }{ }^{\prime} \lambda=\frac{n-2 t}{2 n}, s-\ell<t \leq s & \text { if } \delta=\frac{1}{2}+\frac{k-\ell}{n} \in I_{k, s}^{L} \backslash I_{k, s}^{G}, \\
\text { (iv) } \lambda=\frac{n-2 t}{2 n}, s-\min (\ell, g)<t \leq s & \text { if } \delta=\frac{1}{2}+\frac{k-\ell}{n}=\frac{2 g}{n} \in I_{k, s}^{L} \cap I_{k, s}^{G} .
\end{array}
$$

Proof. Let us sketch the proof. First, existence of the conformally equivariant quantization translates as the triviality of 1-cocycles, that we write down in Lemma 4.10. Then, we characterize precisely the conditions for those 1-cocycles to be trivial in Lemma 4.11 and finally prove that, indeed, they are equivalent to (4.12).

Lemma 4.10. The conformally equivariant quantization exists on $\mathcal{S}_{k, s}^{\delta}$ if and only if, for all $g \leq s$ and $d \leq k-2 s+g$, there is a map $\phi_{d, g} \in \mathcal{D}_{\mathfrak{c e}}\left(\mathcal{S}_{k, s}^{\delta}, \mathcal{S}_{k-d-g, s-g}^{\delta}\right)$ such that

$$
\left[\phi_{d, g}, L_{X_{i}}^{\delta}\right]=\gamma_{d, g}\left(X_{i}\right)
$$


where the right hand side is the corestriction of $\left(\mathcal{L}_{X_{i}}^{\lambda, \mu}-L_{X_{i}}^{\delta}\right) \circ\left(\phi_{d, g-1}+\phi_{d-1, g}\right)$ to the subspace $\mathcal{S}_{k-d-g, s-g}^{\delta}$. It is given by

$$
\gamma_{d, g}\left(X_{i}\right)=\mathbb{G}_{s+1-g}^{k+1-d-g}(\lambda) \phi_{d, g-1}+\mathbb{D}_{s-g}^{k+1-d-g}(\lambda) \phi_{d-1, g},
$$

and for any $2 s^{\prime} \leq k^{\prime}$ the operators $\mathbb{G}_{s^{\prime}}^{k^{\prime}}(\lambda): \mathcal{S}_{k^{\prime}, s^{\prime}}^{\delta} \rightarrow \mathcal{S}_{k^{\prime}-1, s^{\prime}-1}^{\delta}$ and $\mathbb{D}_{s^{\prime}}^{k^{\prime}}(\lambda): \mathcal{S}_{k^{\prime}, s^{\prime}}^{\delta} \rightarrow \mathcal{S}_{k^{\prime}-1, s^{\prime}}^{\delta}$ vanish if and only if $\lambda=\frac{n-2 s^{\prime}}{2 n}$ and $\lambda=\frac{1-\left(k^{\prime}-s^{\prime}\right)}{n}$ respectively.

Proof. The equation (4.13) is simply the projection of equation (2.8) on the space $\mathcal{S}_{k-d-g, s-g}^{\delta}$. Hence, we just have to get the explicit expression of $\gamma_{d, g}\left(X_{i}\right)$. For that, we need to decompose the operator $\mathcal{L}_{X_{i}}^{\lambda, \mu}-L_{X_{i}}^{\delta}$ given in (4.2). Let $R^{s} Q \in \mathcal{S}_{k, s}^{\delta}$, so that $Q$ is in the kernel of $T$. Using the decomposition $p_{i} Q=\pi_{0}\left(p_{i} Q\right)+\frac{2}{\rho_{k-2 s+1,1}} R \partial_{p^{i}} Q$, where $\rho_{k, s}=2 s(n+2(k-s-1))$ is the eigenvalue of $R T$ and $\pi_{0}$ denotes the projection on $\operatorname{ker} T$, we obtain

$$
\begin{aligned}
\left(\mathcal{L}_{X_{i}}^{\lambda, \mu}-L_{X_{i}}^{\delta}\right)\left(R^{s} Q\right)= & 2 s(2 n \lambda+2 s-n) R^{s-1} \pi_{0}\left(p_{i} Q\right) \\
& +\left(2 n \lambda+2(k-1)+2 s \frac{2 n \lambda-2 s-n}{n+2(k-2 s-1)}\right) R^{s} \partial_{p^{i}} Q .
\end{aligned}
$$

Both coefficients are affine functions in $\lambda$ vanishing for the announced values of $\lambda$, and we have $R^{s-1} \pi_{0}\left(p_{i} Q\right) \in \mathcal{S}_{k-1, s-1}^{\delta}$ and $R^{s} \partial_{p^{i}} Q \in \mathcal{S}_{k-1, s}^{\delta}$. Hence, we get the announced expression (4.14) for $\gamma_{d, g}\left(X_{i}\right)$.

Lemma 4.11. Existence of the conformally equivariant quantization on $\mathcal{S}_{k, s}^{\delta}$ is equivalent to: $\gamma_{d, 0}=0, \gamma_{0, g}=0$ or $\gamma_{\ell, \ell}\left(X_{i}\right)$ has no component in $\partial_{i} R^{s-\ell} L_{0}^{\ell-1} T^{s}$ if, respectively, $D^{d}, R^{s-g} G_{0}^{g} T^{s}$ or $R^{s-\ell} \mathcal{L}_{\ell} T^{s}$ is conformally invariant.

Proof. Using the preceding lemma, we know that the conformally equivariant quantization exists if and only if the 1-cocycles $\gamma_{d, g}$ are trivial. But resorting to the proof of Theorem 2.5, this is the case except when there is a conformally invariant operator in $\operatorname{Hom}_{\mathfrak{c e}}\left(\mathcal{S}_{k, s}^{\delta}, \mathcal{S}_{k-d-g, s-g}^{\delta}\right)$. Hence, the previous classification of such operators shows that the only nontrivial 1-cocycle is $\gamma_{d, 0}, \gamma_{0, g}$ or $\gamma_{\ell, \ell}$ if respectively $D^{d}, R^{s-g} G_{0}^{g} T^{s}$ or $R^{s-\ell} \mathcal{L}_{\ell} T^{s}$ is conformally invariant. Since the previously introduced spaces of 1-cocycles $E_{d, 0}$ and $E_{0, g}$ are unidimensional, Proposition 4.8 implies that $\gamma_{d, 0}$ and $\gamma_{0, g}$ are trivial if and only if they vanish. It remains to handle the case of $\gamma_{\ell, \ell}$. We suppose that $R^{s-\ell} \mathcal{L}_{\ell} T^{s}$ is conformally invariant for the shift $\delta$. Let us denote by $C^{\delta^{\prime}}$ the space of 1 -coboundaries $\left[\operatorname{Hom}_{\mathfrak{c e}}\left(\mathcal{S}_{k, s}^{\delta^{\prime}}, \mathcal{S}_{k-\ell, s-\ell}^{\delta^{\prime}}\right), L^{\delta^{\prime}}\right.$ ]. Generically, $\gamma_{\ell, \ell}$ is a 1-coboundary, so it pertains to the space $\lim _{\delta^{\prime} \rightarrow \delta} C^{\delta^{\prime}}=C^{\delta} \oplus \mathbb{R}$. The linear form giving the component in $\partial_{i} R^{s-\ell} L_{0}^{\ell-1} T^{s}$ vanishes precisely on the subspace of 1 -coboundaries $C^{\delta}$, and thus $\gamma_{\ell, \ell}$ is trivial if and only if it pertains to its kernel.

We are ready now to prove the theorem. In each of the four cases in (4.12), i.e. for each critical value $\delta$, we determine the operators $\mathbb{G}_{s^{\prime}}^{k^{\prime}}(\lambda)$ and $\mathbb{D}_{s^{\prime \prime}}^{k^{\prime \prime}}(\lambda)$ which should vanish, so that the previous constraints on the 1-cocycles $\gamma_{d, 0}, \gamma_{0, g}, \gamma_{\ell, \ell}$ are satisfied.

The instances $\delta \in I_{k, s}^{D}$ and $\delta \in I_{k, s}^{G}$ are treated like in the projective case, the concerned space Hom $_{\mathfrak{c e}}$ being unidimensional. In the first case, it exists $d \leq k-2 s$ such that $D^{d}$ is conformally invariant, and Lemma 4.11 implies that $\gamma_{d, 0}=0$. Using equation (4.14), we obtain by induction that $\mathbb{D}_{s}^{h+s}(\lambda)=0$ for some $k-s-d<h \leq k-s$, hence the result (i) of (4.12). If $\delta \in I_{k, s}^{G}$, then it exists $g \leq s$ such that $R^{s-g} G_{0}^{g} T^{s}$ is conformally invariant. Similarly we must have $\gamma_{0, g}=0$ and then, by induction, $\mathbb{G}_{t}^{k-s+t}(\lambda)=0$ for some $s-g<t \leq s$. The result (ii) of (4.12) follows.

The instance $\delta \in I_{k, s}^{L}$ is more involved, and relies, as Lemma 4.2, on independence of monomials $G_{0}^{g} D_{0}^{d} L_{0}^{\ell}$ for different exponents $\ell$. In that case, there exists $\ell \leq s$ such that $R^{s-\ell} \mathcal{L}_{\ell} T^{s}$ 
is conformally invariant and Lemma 4.11 implies that $\gamma_{\ell, \ell}$ must have no component along $\partial_{i} R^{s-\ell} L_{0}^{\ell-1} T^{s}$. We deduce from the formula (4.14) that either $\mathbb{D}_{s-\ell}^{k+1-2 \ell}=0$ or $\phi_{\ell-1, \ell}$ has no component in $R^{s-\ell} G_{0} L_{0}^{\ell-1} T^{s}$. Then, a straightforward induction proves that all the paths in the following diagram (4.15) from Id to $L_{0}^{\ell}$ have a vanishing label on at least one of its arrows. For simplicity we do not write the appropriate powers of $R$ and $T$.

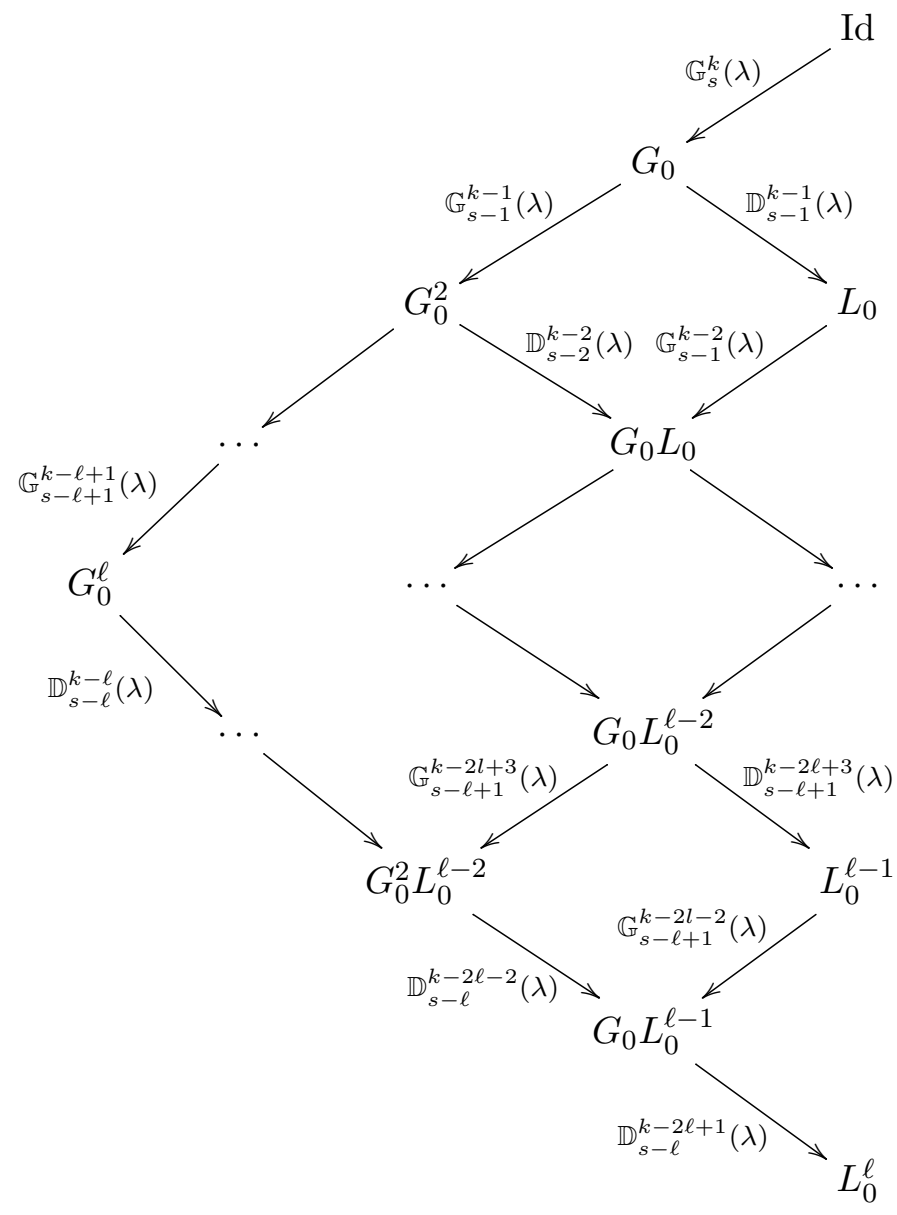

Since $\mathbb{D}_{s-l}^{k-l}(\lambda)$ and $\mathbb{D}_{s}^{k}(\lambda)$ vanish for the same value of $\lambda$, as well as $\mathbb{G}_{s}^{k}(\lambda)$ and $\mathbb{G}_{s}^{k^{\prime}}(\lambda)$, we finally get that, necessarily, $\mathbb{G}_{t}^{k-s+t}(\lambda)=0$ or $\mathbb{D}_{s}^{h+s}(\lambda)=0$ for respectively $s-\ell<t \leq s$ and $k-s-\ell<h \leq k-s$. Hence, we end with the results (iii) and (iii)' of (4.12) for $\delta \in I_{k, s}^{L} \backslash I_{k, s}^{G}$.

At least, we suppose that $\delta \in I_{k, s}^{L} \cap I_{k, s}^{G}$. Then, it exists $g, \ell \leq s$ satisfying the relation $\delta=\frac{1}{2}+\frac{k-\ell}{n}=\frac{2 g}{n}$. We deduce that $\lambda$ must satisfy simultaneously the conditions (ii) and (iii)/(iii)' in (4.12) and that leads precisely to (iv).

The last part of the proof of Theorem 4.9 shows that each $\lambda$ given in (4.12) corresponds to the vanishing of an operator $\mathbb{G}$ or $\mathbb{D}$, i.e. to the vanishing of a certain restriction of $\mathcal{L}^{\lambda, \mu}-L^{\delta}$. The Lemma 3.3 allows then to deduce existence of conformally invariant differential operators on $\lambda$ densities, for each resonance $\lambda$ in (4.12). They organize in two families: the powers of Laplacian $\Delta^{t}: \mathcal{F}^{\frac{n-2 t}{2 n}} \rightarrow \mathcal{F}^{\frac{n+2 t}{2 n}}$ and the operators $\pi_{0} \bar{G}^{h}: \mathcal{F}^{\frac{1-h}{n}} \rightarrow \overline{\mathcal{S}}_{h, 0}^{\frac{1-h}{n}}$ which are built from the operator $\bar{G}=\bar{p}^{i} \partial_{i}$, introduced in (3.5). The latter is dual to the conformal Killing operator $G$ arising in Lemma 4.2. By Weyl's theory of invariants or by the general classification in [2, 3], these are all the conformally invariant differential operators on $\lambda$-densities. Hence, we can reformulate the Theorem 4.9 along the lines of Kroeske's paradigm. Notice that it is done à posteriori, contrary to the Theorem 2.5. 
Theorem 4.12. The conformally equivariant quantization $\mathcal{Q}^{\lambda, \mu}: \mathcal{S}_{k, s}^{\delta} \otimes \mathcal{F}^{\lambda} \rightarrow \mathcal{F}^{\mu}$ exists and is unique except when there is a conformally invariant differential operator: $\mathcal{S}_{k, s}^{\delta} \rightarrow \mathcal{S}_{k-l, s-r}^{\delta}$. Then, $\mathcal{Q}^{\lambda, \mu}$ exists if and only if there is a conformally invariant differential operator from $\mathcal{F}^{\lambda}$ to sections of a homogeneous bundle $\mathcal{V}$, whose principal symbol lies in $\mathcal{S}_{h, t}^{-\lambda} \otimes \Gamma(\mathcal{V})$ with $\mathcal{D}_{\mathfrak{c} \mathfrak{e}}\left(\mathcal{S}_{k, s}^{\delta}, \mathcal{S}_{h, t}^{\delta}\right) \neq\{0\}$ and $\mathcal{D}_{\mathfrak{c e}}\left(\mathcal{S}_{k-l, s-r}^{\delta}, \mathcal{S}_{h, t}^{\delta}\right)=\{0\}$.

Silhan provides in [23] an explicit construction for the conformally equivariant quantization when the shift $\delta$ is not one of the critical values (4.11), and also two alternative constructions in the critical cases. In the next two propositions, we prove that they allow to handle all the remaining cases of existence, when $(\lambda, \lambda+\delta)$ is a resonance (4.12).

Proposition 4.13. In the three cases (ii), (iii), (iv) of (4.12), the operator $\Delta^{t}: \mathcal{F}^{\lambda} \rightarrow \mathcal{F}^{\lambda+\frac{2 t}{n}}$ is conformally invariant. The commutative diagram

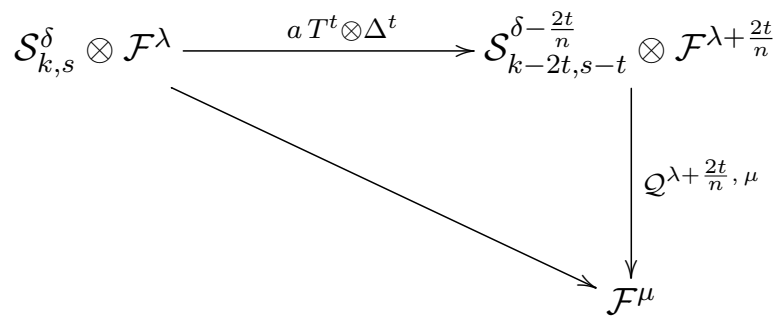

defines then a conformally equivariant quantization on $\mathcal{S}_{k, s}^{\delta}$ for a well-chosen constant $a \in \mathbb{R}$.

Proof. The operator $a T^{t}$ above is conformally invariant, and $a$ can be chosen such that the equality $a R^{t} T^{t}=\mathrm{Id}$ holds on $\mathcal{S}_{k, s}^{\delta}$, ensuring the good normalization. Moreover, the conformally equivariant quantization $\mathcal{Q}^{\lambda+\frac{2 t}{n}, \mu}$ is well defined on $\mathcal{S}_{k-2 t, s-t}^{\delta-\frac{2 t}{n}}$ by Theorem 4.6.

Let us introduce a refinement of the filtration of $\mathcal{D}^{\lambda, \mu}$, which already appears in [7]. Namely, we denote by $\mathcal{D}_{k, s}^{\lambda, \mu}$ the subspace of $\mathcal{D}_{k}^{\lambda, \mu}$ given by the image of $\bigoplus_{0 \leq s-r \leq k-l} \mathcal{S}_{l, r}^{\delta}$ under the normal ordering. The expression (4.2) of the action of $\mathfrak{c f}$ shows that $\mathcal{D}_{k, s}^{\lambda, \mu}$ is in fact a $\mathfrak{c f}$-submodule of $\mathcal{D}_{k}^{\lambda, \mu}$. The subspaces $\mathcal{S}_{l, r}^{\delta}$ arising in its definition are characterized equivalently by $\mathcal{D}_{\mathfrak{c e}}\left(\mathcal{S}_{k, s}^{\delta}, \mathcal{S}_{l, r}^{\delta}\right) \neq 0$ or, with the notation of Lemma 4.10, by their presence in the following tree, analogous to previous ones (4.6) and (4.15),

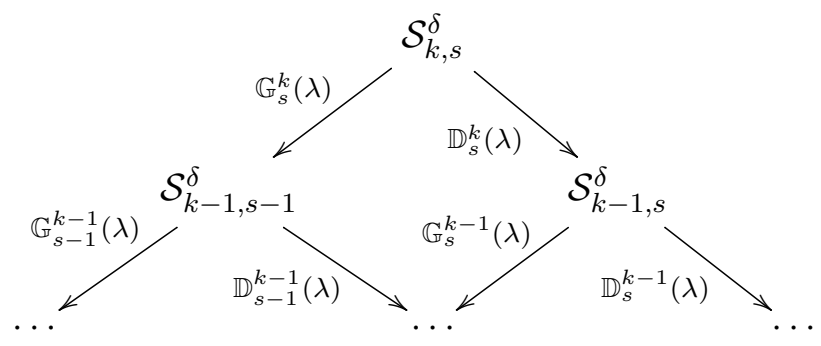

We can restrict the differential operators obtained by morphism $\left(\iota_{\alpha}\right)^{j}$, defined in (3.6), to traceless tensors. The resulting morphism of $\mathfrak{c f}$-modules $\left(\iota_{\alpha}\right)_{0}^{j}: \mathcal{D}_{k, s}^{\lambda, \mu} \rightarrow \mathcal{D}_{k-h}^{\lambda, \mu}\left(\overline{\mathcal{S}}_{h, 0}^{\lambda}, \mathcal{F}^{\mu}\right)$ has clearly a nontrivial kernel, which is equal to $\mathcal{D}_{j-1+r, r}^{\lambda, \mu} \supset \mathcal{D}_{j-1}^{\lambda, \mu}$ if $r=\min (s, j-1)$. This leads to the following result, similar to Proposition 3.4 dealing with the projective case. 
Proposition 4.14. In the two cases (i) and (iii) of (4.12), the operator $\pi_{0} \bar{G}^{h}: \mathcal{F}^{\lambda} \rightarrow \bar{S}_{h, 0}^{\lambda}$ is conformally invariant. The commutative diagram

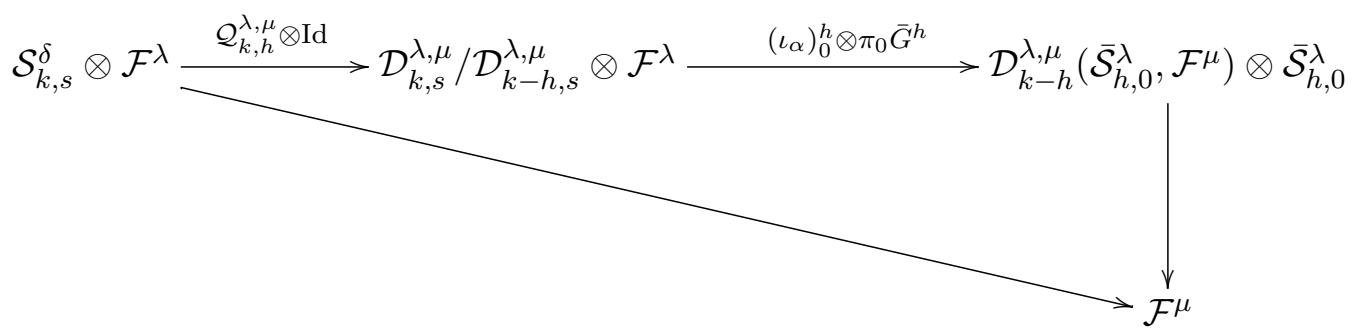

defines then a conformally equivariant quantization on $\mathcal{S}_{k, s}^{\delta}$.

Proof. In view of the definition of $h$, there is no obstruction to the existence of the partial conformally equivariant quantization $\mathcal{Q}_{k, h}^{\lambda, \mu}$. Using the conformal invariance of $\pi_{0} \bar{G}^{h}$ and of $\left(\iota_{\alpha}\right)_{0}^{h}$, we get the result.

\subsection{Example: conformally equivariant quantization of symbols of degree 3}

The conformally equivariant quantization as been explicitly determined up to order 3 in the momenta by Loubon Djounga in [18]. We give here for each submodule $\mathcal{S}_{k, s}^{\delta}$, with $k \leq 3$, the conformally invariant operators acting on it, the corresponding critical values of the shift $\delta$, the resonances $(\lambda, \lambda+\delta)$ and the associated conformally invariant operators on $\mathcal{F}^{\lambda}$.

\begin{tabular}{|c||c|c|c|c|}
\hline Space & Op. Inv. on $\mathcal{S}_{k, s}^{\delta}$ & $\delta$ & $\lambda$ & Op. Inv. on $\mathcal{F}^{\lambda}$ \\
\hline $\mathcal{S}_{1}^{\delta}$ & $D$ & 1 & 0 & $\bar{G}$ \\
\hline $\mathcal{S}_{2,0}^{\delta}$ & $D$ & $\frac{n+2}{n}$ & $-\frac{1}{n}$ & $\bar{G}^{2}$ \\
& $D^{2}$ & $\frac{n+1}{n}$ & $-\frac{1}{n}, 0$ & $\bar{G}^{2}, \bar{G}$ \\
\hline $\mathcal{S}_{2,1}^{\delta}$ & $G_{0} T$ & $\frac{2}{n}$ & $\frac{n-2}{2 n}$ & $\Delta$ \\
& $L_{0} T$ & $\frac{n+2}{2 n}$ & $\frac{n-2}{2 n}, 0$ & $\Delta, \bar{G}$ \\
\hline $\mathcal{S}_{3,0}^{\delta}$ & $D$ & $\frac{n+4}{n}$ & $-\frac{2}{n}$ & $\bar{G}^{3}$ \\
& $D^{2}$ & $\frac{n+3}{n}$ & $-\frac{2}{n},-\frac{1}{n}$ & $\bar{G}^{3}, \bar{G}^{2}$ \\
& $D^{3}$ & $\frac{n+2}{n}$ & $-\frac{2}{n},-\frac{1}{n}, 0$ & $\bar{G}^{3}, \bar{G}^{2}, \bar{G}$ \\
\hline $\mathcal{S}_{3,1}^{\delta}$ & $R D T$ & $\frac{n+2}{n}$ & $-\frac{1}{n}$ & $\bar{G}^{2}$ \\
& $G_{0} T$ & $\frac{2}{n}$ & $\frac{n-2}{2 n}$ & $\Delta$ \\
& $\mathcal{L}_{1} T$ & $\frac{n+2}{n}$ & $\frac{n-2}{2 n},-\frac{1}{n}, 0$ & $\Delta, \bar{G}^{2}, \bar{G}$ \\
\hline
\end{tabular}

Let $\delta$ be one of the above critical values. If we consider the conformally equivariant quantization on the whole space $\mathcal{S}_{3}^{\delta} \oplus \mathcal{S}_{2}^{\delta} \oplus \mathcal{S}_{1}^{\delta} \oplus \mathcal{S}_{0}^{\delta}$, as in [18], then it will exist only for the values of $\lambda$ appearing in each row where $\delta$ is present. Thus, we recover precisely the result of [18].

\section{Prospects}

We have proven in the setting of IFFT-equivariant quantization, introduced in [4], that unique existence of the quantization map is lost if and only if there is an invariant differential operator 
on the space of symbols. The work of Cap and Silhan [6] allows to trivially extend this result to IFFT-equivariant quantization with values in differential operators acting on homogeneous irreducible bundles. It remains then to characterize when equivariant quantization exists but is not unique. We have done it only for projectively and conformally equivariant quantizations, with values in $\mathcal{D}^{\lambda, \mu}$, in terms of existence of an invariant differential operator on the module $\mathcal{F}^{\lambda}$ of $\lambda$-densities. In the light of these both examples we propose the following method to link resonances with invariant operators on the source space (e.g. $\mathcal{F}^{\lambda}$ ).

- To each invariant differential operator acting on the space of symbols corresponds a nontrivial 1-cocycle $\gamma$.

- The latter obstructs the existence of the equivariant quantization except for a finite number of values of $\lambda$.

- For those values, a certain restriction of the operator $\mathcal{L}^{\lambda, \mu}-L^{\delta}$ vanishes.

- An invariant differential operator on the source space can then be built, and we obtain all of them in this way.

- An equivariant quantization can be design from those invariant operators.

We hope that this will lead to the proof of the point (2) in the following conjecture, the point (1) being Theorem 2.6.

Conjecture 5.1. Let $\mathfrak{g}$ be an IFFT-algebra, $\mathcal{V}, \mathcal{W}$ be irreducible homogeneous bundles and $\mathcal{B}$ a submodule of $\mathcal{S}_{k}^{\delta}(\mathcal{V}, \mathcal{W})$. The $\mathfrak{g}$-equivariant quantization $\mathcal{B} \otimes\left(\Gamma(\mathcal{V}) \otimes \mathcal{F}^{\lambda}\right) \longrightarrow \Gamma(\mathcal{W}) \otimes \mathcal{F}^{\mu}$ satisfies the following properties:

1) it does not exist or is not unique if and only if, for $1 \leq l \leq k$, there is a $\mathfrak{g}$-invariant differential operator: $\mathcal{B} \rightarrow \mathcal{C}$, with $\mathcal{C}$ a submodule of $\mathcal{S}_{k-l}^{\delta}(\mathcal{V}, \mathcal{W})$,

2) for such $\delta$, the $\mathfrak{g}$-equivariant quantization exists if and only if there is a $\mathfrak{g}$-invariant differential operator on $\Gamma(\mathcal{V}) \otimes \mathcal{F}^{\lambda}$, whose principal symbol lies in $\mathcal{A}$ and satisfies the both conditions $\mathcal{D}_{\mathfrak{g}_{-1} \oplus \mathfrak{g}_{0}}(\mathcal{B}, \mathcal{A}) \neq 0$ and $\mathcal{D}_{\mathfrak{g}_{-1} \oplus \mathfrak{g}_{0}}(\mathcal{C}, \mathcal{A})=0$.

The next step will be to generalize such a result for $\mathfrak{g}$-invariant bidifferential pairings, which are bidifferential operators $\Gamma(\mathcal{V}) \otimes \Gamma(\mathcal{W}) \rightarrow \Gamma(\mathcal{T})$, where $\mathcal{V}, \mathcal{W}, \mathcal{T}$ are irreducible homogeneous bundles. Let us discuss the peculiar case of the generalized transvectants or Rankin-Cohen brackets. It has been proved in [22] that there exists a unique conformally invariant bidifferential operator of order $2 k$ acting on $\lambda$ - and $\mu$-densities, $B_{2 k}^{\lambda, \mu}: \mathcal{F}^{\lambda} \otimes \mathcal{F}^{\mu} \rightarrow \mathcal{F}^{\lambda+\mu+\frac{2 k}{n}}$, if and only if the weights $\lambda$ and $\mu$ do not pertain to the set of exceptional values $\left\{\frac{n-2 k}{2 n}, \ldots, \frac{n-2}{2 n}\right\} \cup\left\{\frac{2-2 k}{n}, \ldots, 0\right\}$. Remarkably they precisely coincide with the resonances of the conformally equivariant quantization. For those exceptional values, we can construct new generalized transvectants from the conformally invariant operators $\Delta^{\ell}$ and $G_{0}^{g}$. Explicitly, they are given by $B_{2(k-\ell)}^{\lambda+\frac{2 \ell}{n}, \mu} \circ\left(\Delta^{\ell} \otimes \mathrm{Id}\right)$ if $\ell \leq k$ and $\lambda \in\left\{\frac{n-2 k}{2 n}, \ldots, \frac{n-2}{2 n}\right\}$, and by $\mathcal{Q}^{\lambda, \mu} \circ\left(G_{0}^{g} \otimes \mathrm{Id}\right)$ if $g \leq 2 k-1, \mu$ is generic and $\lambda \in\left\{\frac{2-2 k}{n}, \ldots, 0\right\}$. A tight link between bidifferential and differential invariant operators shows up one more time. The same kind of idea is used in [1], where new conformally invariant trilinear forms on tensor densities are built from the invariant operators $\Delta^{\ell}$. To conclude, Kroeske's paradigm definitely asks for deeper investigations.

\section{Acknowledgements}

It is a pleasure to acknowledge Christian Duval, Pierre Mathonet and Valentin Ovsienko for fruitful discussions and the referees for suggesting numerous improvements. I thank the Luxembourgian NRF for support via the AFR grant PDR-09-063. 


\section{References}

[1] Beckmann R., Clerc J.L., Singular invariant trilinear forms and covariant (bi-)differential operators under the conformal group, J. Funct. Anal. 262 (2012), 4341-4376.

[2] Boe B.D., Collingwood D.H., A comparison theory for the structure of induced representations, J. Algebra 94 (1985), 511-545.

[3] Boe B.D., Collingwood D.H., A comparison theory for the structure of induced representations. II, Math. Z. 190 (1985), 1-11.

[4] Boniver F., Mathonet P., IFFT-equivariant quantizations, J. Geom. Phys. 56 (2006), 712-730, math.RT/0109032.

[5] Boniver F., Mathonet P., Maximal subalgebras of vector fields for equivariant quantizations, J. Math. Phys. 42 (2001), 582-589, math.DG/0009239.

[6] Cap A., Silhan J., Equivariant quantizations for AHS-structures, Adv. Math. 224 (2010), 1717-1734, arXiv:0904.3278.

[7] Duval C., Lecomte P., Ovsienko V., Conformally equivariant quantization: existence and uniqueness, Ann. Inst. Fourier (Grenoble) 49 (1999), 1999-2029, math.DG/9902032.

[8] Duval C., Ovsienko V., Conformally equivariant quantum Hamiltonians, Selecta Math. (N.S.) 7 (2001), 291-320, math.DG/9801122.

[9] Duval C., Ovsienko V., Projectively equivariant quantization and symbol calculus: noncommutative hypergeometric functions, Lett. Math. Phys. 57 (2001), 61-67, math.QA/0103096.

[10] Eastwood M., Slovák J., Semiholonomic Verma modules, J. Algebra 197 (1997), 424-448.

[11] Eastwood M.G., Rice J.W., Conformally invariant differential operators on Minkowski space and their curved analogues, Comm. Math. Phys. 109 (1987), 207-228, Erratum, Comm. Math. Phys. 144 (1992), 213.

[12] Fuks D.B., Cohomology of infinite-dimensional Lie algebras, Contemporary Soviet Mathematics, Consultants Bureau, New York, 1986.

[13] Kobayashi S., Nagano T., On filtered Lie algebras and geometric structures. I, J. Math. Mech. 13 (1964), 875-907.

[14] Kroeske J., Invariant bilinear differential pairings on parabolic geometries, Ph.D. thesis, University of Adelaide, 2008, arXiv:0904.3311.

[15] Kroeske J., Invariant differential pairings, Acta Math. Univ. Comenian. (N.S.) 77 (2008), 215-244, math.DG/0703866.

[16] Lecomte P.B.A., On the cohomology of $\operatorname{sl}(m+1, \mathbb{R})$ acting on differential operators and $\operatorname{sl}(m+1, \mathbb{R})$ equivariant symbol, Indag. Math. (N.S.) 11 (2000), 95-114, math.DG/9801121.

[17] Lecomte P.B.A., Ovsienko V.Y., Projectively equivariant symbol calculus, Lett. Math. Phys. 49 (1999), 173-196, math.DG/9809061.

[18] Loubon Djounga S.E., Modules of third-order differential operators on a conformally flat manifold, J. Geom. Phys. 37 (2001), 251-261.

[19] Mathonet P., Radoux F., Cartan connections and natural and projectively equivariant quantizations, J. Lond. Math. Soc. (2) 76 (2007), 87-104, math.DG/0606556.

[20] Mathonet P., Radoux F., Existence of natural and conformally invariant quantizations of arbitrary symbols, J. Nonlinear Math. Phys. 17 (2010), 539-556, arXiv:0811.3710.

[21] Nikitin A.G., Prilipko A.I., Generalized Killing tensors and the symmetry of the Klein-Gordon-Fock equation, Preprint no. 90.23, Institute of Mathematics, Kyiv, 1990, 59 pages, math-ph/0506002.

[22] Ovsienko V., Redou P., Generalized transvectants-Rankin-Cohen brackets, Lett. Math. Phys. 63 (2003), 19-28, math.DG/0104232.

[23] Silhan J., Conformally invariant quantization - towards complete classification, arXiv:0903.4798.

[24] Weyl H., The classical groups. Their invariants and representations, Princeton Landmarks in Mathematics, Princeton University Press, Princeton, NJ, 1997.

[25] Wünsch V., On conformally invariant differential operators, Math. Nachr. 129 (1986), 269-281. 